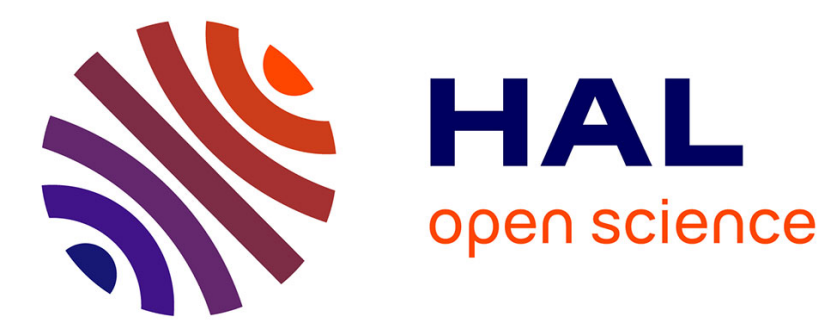

\title{
Spinal sensory neurons project onto hindbrain to stabilize posture and enhance locomotor speed
}

Ming-Yue Wu, Martin Carbó-Tano, Olivier Mirat, Francois-Xavier Lejeune, Julian Roussel, Feng Quan, Kevin Fidelin, Claire Wyart

\section{To cite this version:}

Ming-Yue Wu, Martin Carbó-Tano, Olivier Mirat, Francois-Xavier Lejeune, Julian Roussel, et al.. Spinal sensory neurons project onto hindbrain to stabilize posture and enhance locomotor speed. Current Biology - CB, 2021, 10.1016/j.cub.2021.05.042 . hal-03334718

\section{HAL Id: hal-03334718 https://hal.science/hal-03334718}

Submitted on 4 Sep 2021

HAL is a multi-disciplinary open access archive for the deposit and dissemination of scientific research documents, whether they are published or not. The documents may come from teaching and research institutions in France or abroad, or from public or private research centers.
L'archive ouverte pluridisciplinaire HAL, est destinée au dépôt et à la diffusion de documents scientifiques de niveau recherche, publiés ou non, émanant des établissements d'enseignement et de recherche français ou étrangers, des laboratoires publics ou privés. 
Wu et al. revised manuscript to Current Biology

\section{Format}

Article

Title

Spinal sensory neurons project onto hindbrain to stabilize posture and enhance locomotor speed

\section{Authors}

Ming-Yue $\mathrm{Wu}^{1 *}$, Martin Carbó-Tano ${ }^{1 *, \#,}$ Olivier Mirat ${ }^{1}$, Francois-Xavier Lejeune ${ }^{1}$, Julian Roussel ${ }^{1}$, Feng Quan ${ }^{1}$, Kevin Fidelin ${ }^{1,2}$, Claire Wyart ${ }^{1, \#}$

\section{Affiliations}

1 Sorbonne Université, Institut du Cerveau (ICM), Inserm U 1127, CNRS UMR 7225, 75013, Paris, France

2 Present address: Friedrich Miescher Institute for Biomedical Research, 4058 Basel, Switzerland

${ }^{*}$ equal contributors

\# Correspondence: claire.wyart@icm-institute.org; martin.carbotano@icm-institute.org; Address: ICM, 47 boulevard de l'Hôpital, 75013 Paris.

\section{SUMMARY}

In the spinal cord, cerebrospinal fluid-contacting neurons (CSF-cNs) are GABAergic interoceptive sensory neurons that detect spinal curvature via a functional coupling with the Reissner fiber. This mechanosensory organ has recently been found involved in spine morphogenesis and postural control. In zebrafish, CSF-cNs project an ascending and ipsilateral axon reaching two to six segments away. Rostralmost CSF-cNs send their axons into the hindbrain, a brain region containing motor nuclei and reticulospinal neurons (RSNs), which send descending motor commands to spinal circuits. Until now, the synaptic connectivity of CSF-cNs has only been investigated in the spinal cord where they synapse onto motor neurons and excitatory premotor interneurons. The identity of CSF$\mathrm{cN}$ targets in the hindbrain and the behavioral relevance of these sensory projections from spinal cord to hindbrain are unknown. Here, we provide anatomical and molecular evidence that rostralmost CSF-cNs synapse onto the axons of large pontine RSNs including the Mauthner cells and early born $\mathrm{Ch} \times 10^{+}$neurons. Functional anatomy and 
Wu et al. revised manuscript to Current Biology

optogenetic-assisted mapping reveal that rostral CSF-cNs also synapse onto the soma and dendrites of cranial motor neurons innervating hypobranchial muscles. During acousto-vestibular stimulus evoked escapes, ablation of rostralmost CSF-cNs results in a defective escape response with a decreased C-bend amplitude, lower speed and a deficient postural control. Our study demonstrates that spinal sensory feedback enhances speed and stabilizes posture, and reveals a novel spinal gating mechanism acting on the output of descending commands sent from the hindbrain to the spinal cord.

\section{Keywords}

Cerebrospinal fluid (CSF), cerebrospinal fluid-contacting neurons (CSF-cNs), polycystic kidney disease 2 like 1 (PKD2L1), mechanosensory feedback, hindbrain, spinal cord, locomotion, speed, posture, reticulospinal neurons (RSNs), cranial motor neurons.

\section{eTOC}

Cerebrospinal fluid-contacting neurons are mechanosensory cells that detect spinal curvature. Wu et al. show here that rostralmost CSF-cNs synapse in the hindbrain onto cranial motor neurons and the descending axons of reticulospinal neurons, and enhance speed and power as well as postural control during active locomotion.

\section{Highlights}

- Cerebrospinal fluid-contacting neurons (CSF-cNs) in rostral spinal cord form inhibitory synapses onto cranial motor neurons

- Rostral CSF-cNs synapse onto descending axons of reticulospinal neurons

- CSF-cN sensory feedback in the rostral spinal cord enhance speed and power of locomotion

- Rostral CSF-cNs projecting to the hindbrain contribute to postural control

\section{INTRODUCTION}

During locomotion in vertebrates, spinal "central pattern generators" (CPGs) produce rhythmic motor output to coordinate muscle contraction throughout the body $[1,2]$. Reticulospinal neurons (RSNs) in the hindbrain receive inputs from higher brain motor areas and in turn, send descending commands to spinal CPGs $[3,4]$. Sensory stimulation such as an acousto-vestibular (AV) or a noxious stimulus elicits an escape response via the activation of RSNs through polysynaptic pathways [5-8]. The recruitment of different RSNs correlates with initiation $[9,10]$, maintenance [11-13] and stop of locomotion [14- 
16]. Spinal CPGs controlling axial and limb muscles must act in coordination with hindbrain motor nuclei that control movements of the eyes [17,18] and head [19-22]. In fish, motor neurons (MNs) from the occipital/pectoral $(\mathrm{Oc} / \mathrm{Pec})$ column located in the posterior hindbrain and rostral spinal cord controls the contractions of occipital and pectoral fin muscles [22-25].

Although not necessary to generate oscillatory locomotor activity, sensory feedback from the periphery is critical for modulating the power and setting the timing of motor output during active locomotion [26-29]. In addition to classical proprioceptive sensory pathways located in the peripheral nervous system, recent investigations have revealed a novel and highly conserved mechanosensory system in the spinal cord [30-36]. Cerebrospinal fluidcontacting neurons (CSF-cNs) form together with the Reissner fiber [31,32,37] a sensory system that detects spinal curvature $[30,34,35,38,39]$ and in turn, shapes spine morphogenesis [34,40-43]. CSF-cN mechanosensory function relies on the transient receptor potential channel PDK2L1 $[30,34]$. In the vertebrate spinal cord, the pkd2/1 promoter specifically drives expression in CSF-CNs [44-46]. Using this promoter, we found that the genetic blockage of neurotransmission in CSF-cNs reduces locomotor frequency therefore impacting speed [30] and hampers postural control during AV escapes [39], demonstrating the contribution of this intraspinal mechanosensory feedback to locomotion and posture. However, the underlying circuit that could mediate such effects on locomotor frequency and posture is unknown.

The connectivity map of this spinal sensory system is critical to understand its physiological functions. In larval zebrafish, CSF-cNs send ipsilaterally their axon ascending from two to six segments [46]. In the spinal cord, we showed that CSF-CNs synapse onto the premotor interneurons $\mathrm{V} 0-\mathrm{v}$ [44] involved in slow locomotion [47-50], onto the primary motor neurons (CaP) [39] that innervate the entire ventral musculature [51,52], and onto the primary sensory interneurons (CoPA) [39] involved in sensory-motor gating $[53,54]$. We found that optogenetic activation of the rostral but not caudal CSF-cNs disrupted the spinal antero-posterior propagation of motor activity, suggesting a pivotal role of rostral CSF-cNs in rostrocaudal spinal propagation of motor activity [44]. Interestingly, although rostralmost CSF-cNs with their soma in segments 4-9 densely 
project to the hindbrain [46], their targets and specific contribution to locomotion and posture are unknown.

Here, we took advantage of the transparency and genetic tractability of zebrafish larva to study the connectivity and physiological relevance of rostral CSF-cNs. We found that rostralmost CSF-cNs form synapses onto the soma and dendrite of occipital MNs in the caudal hindbrain. We then confirmed using optogenetically-assisted connectivity mapping that these anatomical projections lead to GABAergic monosynaptic currents in occipital MNs. We also discovered that CSF-cNs formed inhibitory synapses onto descending axons of RSNs involved in producing fast escape behaviors. The apposition of pre- and post-synaptic markers on presynaptic boutons of RSNs suggest that CSF-cNs provide presynaptic inhibition on command neurons. Accordingly, the ablation of rostralmost CSFcNs strikingly reduced locomotor speed and power, as well as increased rolling during AV escapes. Overall, our work reveals that 1 ) rostralmost CSF-cNs extensively form synapses onto hindbrain motor neurons and spinal projecting neurons, and 2) these cells control power, speed and active posture during ongoing locomotion. A large body of literature showed that sensory inputs modulate command neurons in the hindbrain via typically 2 - 4 synapses [7,55-57]. Here we show that mechanosensory feedback from spinal cord can directly modulate the output of descending commands from the hindbrain, opening new paths for investigation of active sensorimotor integration impacting command output in motion.

\section{RESULTS}

\section{Rostral spinal CSF-cNs synapse onto hindbrain occipital/pectoral motor column}

In order to identify the targets of rostral CSF-cNs in the hindbrain, we combined fluorescent transgenic lines labeling CSF-cNs using the specific pkd2/1 promoter $[30,39,44]$ with lines targeting other genetically-defined neuronal populations. We first investigated whether rostral CSF-cNs contact cranial and pectoral motor neurons (MNs) to coordinate head and tail movements using double transgenic Tg(pkd2/1:tagRFP;parga ${ }^{m n 2 E t: G F P)}$ larvae [59] (Figure 1A). In the caudal hindbrain, CSFcNs projected axons ventrally towards the Oc/Pec motor column, but not to the dorsally- 
located vagal motor column (Figure 1A2, 1A3). CSF-cNs projections formed a thin oblique reticular lamina in the caudal hindbrain (Figure 1A3). With the cranial MNs serving as landmarks [21], we observed that CSF-cN axons reached the anterior portion of Rhombomere 8 (Figure 1A4). CSF-cN axons formed numerous boutons on the large soma of primary MNs located in the lateral Oc/Pec column (Figure 1A5, 1A5', 1A5", arrows, and Video S1). We also employed the Tg(zCREST2-hsp70:GFP) transgenic line that contains a subset of occipital MNs in the rostral hindbrain together with abductor pectoral MNs innervating fin muscles [60]. In Tg(pkd2/1:tagRFP;zCREST2-hsp70:GFP) larvae, CSF-cN axons formed a basket-like synaptic structure onto large MNs located lateral in the Oc/Pec column (Figure 1B1, B1') precisely in the same location as observed in the $T g$ (parga ${ }^{m n 2 E t}$ GFP) line (Figure 1A5, 1A5', and 1A5'). In addition, the Oc/Pec MNs protruded their dendrites towards the lateral hindbrain margin where CSF-cN axons project and form en passant boutons on the MN dendrites (Figure 1B2, 1B2'; and Video S2). Since the pectoral MNs located in the hindbrain are medially located within the hindbrain-spinal cord boundary and have many similarities with secondary MNs $[24,58,61]$, the MNs receiving CSF-cNs boutons most likely belong to the occipital MN pool. We confirm the $\mathrm{MN}$ identity by dye filling of single occipital $\mathrm{MN}$ in the triple transgenic Tg(pargamn2Et:GFP; $\alpha$-actin:GAL4;UAS:ChR2-YFP) larvae (Figure 1C1, 1C2, see STAR Methods). The axons of the single MN projected ventrally to the hypobranchial region to innervate the whole axial muscle sternohyoideus (Figure 1C2', 1C2"') [62].

We further demonstrated that CSF-cNs synapse onto the occipital MNs by combining in vivo optogenetic activation of CSF-cNs using Channelrhodopsin2 (ChR2) and whole-cell recordings of MNs using the triple transgenic Tg(pkd2/1:GAL4;UAS:ChR2-mCherry;

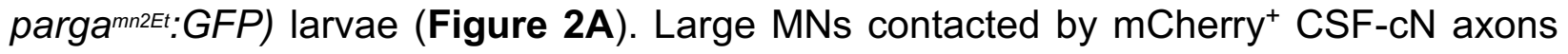
were rostralmost and lateral (Figure 2B). Dye filling during the recording showed that the MN dendrites protruded into the lateral hindbrain (Figure 2B1, 2B1'). At resting membrane potential, these occipital MNs showed rhythmic bursting activity (Figure 2C). A cocktail of $10 \mu \mathrm{M}$ AP5 and $10 \mu \mathrm{M}$ CNQX applied in the bath blocked excitatory inputs and isolated putative inhibitory synaptic currents. These occipital MNs showed characteristic phasic action potential firing in response to current injection (Figure 2D) [63]. In our conditions, as we previously showed [39,44], $5 \mathrm{~ms}$ blue light pulses reliably 
induced single spikes in CSF-cNs expressing ChR2. Upon such optical activation of CSFcNs, large inhibitory postsynaptic currents (IPSCs) were recorded without failure in occipital MNs (Figure 2E1, 2F, 2I, mean \pm SEM: $-39.02 \pm 2.1 \mathrm{pA}, \mathrm{n}=5$ out of 5 cells). As the optically-induced spiking typically occurs in CSF-cNs within $\sim 5 \mathrm{~ms}$ [44], the delay of the light-induced response of $5.9 \pm 0.1 \mathrm{~ms}$ relative to the beginning of the light pulse (Figure 2H) was consistent with a monosynaptic IPSC. Unlike the CaP MNs in spinal cord [39], we did not observe short-term synaptic depression in occipital MNs upon optical stimulation of CSF-cNs at $10-25 \mathrm{~Hz}(20 \mathrm{~Hz}$ light pulses shown in Figure 2E2). The lightinduced responses were abolished by bath application of $10 \mu \mathrm{M} \mathrm{GABA}_{\mathrm{A}}$ receptor antagonist gabazine (Figure 2G, $\mathrm{n}=2$ cells), indicating that IPSCs were mediated by ionotropic $\mathrm{GABA}_{\mathrm{A}}$ receptors. Furthermore, the light-induced IPSCs were characterized by short rise time (1.62 $\pm 0.13 \mathrm{~ms}$ ) and short time decay ( $\mathrm{T}: 15.10 \pm 0.92 \mathrm{~ms}$ ) (Figure 2J and 2K), consistent with $\mathrm{GABA}_{\mathrm{A}}$ receptor-mediated IPSCs [39].

\section{CSF-cNs synapse onto descending axons of large reticulospinal neurons}

We next examined whether the ascending CSF-cN axons could innervate the reticulospinal system. Different spinal projecting neurons are recruited as a function of locomotor speed and direction [56,64-69]. In teleost, the Mauthner cells are two giant hindbrain RSNs that are indirectly recruited upon tactile [70,71], visual [68], or auditory/vestibular [72] stimuli and send commissural axons descending in the spinal cord in order to promptly initiate on the contralateral side the C-start escape response [70,73,74]. We used Tg(pkd2/1:tagRFP;Tol056:GFP) larvae in which the Mauthner cells express GFP [76] to show that the CSF-cN axons formed numerous varicosities onto the descending axon of the Mauthner cells in the medial longitudinal fasciculus (MLF) (Figure 3A1-3A6). Mauthner cell has an unusual wide-caliber and myelinated axon in which synaptic release and saltatory conduction occur within large presynaptic boutons deprived of myelination [75] (Figure 3B1-3B3). In search for evidence of synapses from CSF-cNs onto the Mauthner cell axon, we injected the construct UAS:synaptophysin$m$ Cherry in double transgenic $T g$ (pkd2/1:GAL4;Tol056:GFP) eggs. This approach enables to locate all presynaptic sites with Synaptophysin-mCherry in single mosaicallylabeled CSF-cN to test whether CSF-cNs form presynaptic terminals in close proximity with the large presynaptic boutons of Mauthner cell axon (Figure 3B1-3B3). We found 
Wu et al. revised manuscript to Current Biology

that only ventral CSF-cN formed presynaptic terminals onto the descending axon of the Mauthner cells (Figure 3B1, Figure S1). Presynaptic sites of ventral CSF-cNs precisely contacted the large presynaptic boutons of the Mauthner cell (Figure 3B2, 3B3), suggesting CSF-cNs exert presynaptic inhibition and can therefore modulate the output of this major spinal projecting neuron. Conversely, dorsolateral CSF-cNs rarely projected to the MLF where the Mauthner cell axons are located, and extended instead laterally to the lateral fasciculus tract (Figure 3C, Figure S1).

We next investigated whether $\mathrm{Ch} \times 10^{+} \mathrm{RSN}$ in the hindbrain may be modulated by CSFcNs. Early-born glutamatergic $\mathrm{Ch} \times 10^{+} \mathrm{RSNs}$ send their axons into the MLF and contribute to escape and turning behaviors, while later-born $\mathrm{Chx} 10^{+} \mathrm{RSNs}$ project their axons in the lateral fasciculus contributing to slow locomotion [69]. We observed in Tg(pkd2/1:tagRFP; $\operatorname{ch} \times 10: G F P)$ larvae [77] that CSF-cN axons formed varicosities on the Chx10+ descending axons in the MLF as well as in the lateral fasciculus (Figure 3D). We labeled presynaptic sites with Synaptophysin-mCherry in sparsely-labeled CSF-cNs as described above using Tg(pkd2/1:GAL4; chx10:GFP). Ventral CSF-cNs formed numerous synapses onto the descending axon of the $\mathrm{Ch} \times 10^{+} \mathrm{RSNs}$ in the MLF (Figure 3E, $\mathrm{n}=14$ out of 16 ventral CSF-cNs) while dorsolateral CSF-cNs mainly formed synapses onto the axon of $\mathrm{Ch \times 10^{+ }}$ neurons in the lateral fasciculus in the spinal cord (Figure 3F, $\mathrm{n}=20$ out of 20 dorsolateral CSF-cNs). We then confirmed that CSF-cN varicosities form inhibitory synapses onto $c h \times 10^{+}$neurons by injecting the construct pT2M-UAS:mCherry-zGephyrinaP1 in Tg(pkd211:GFP;chx10:GAL4) eggs [78]. We observed that the lateral fasciculus, $\mathrm{GFP}^{+}$CSF-cN varicosities overlapped with mCherry-zGephyrin ${ }^{+}$postsynaptic densities indicative of inhibitory synapses onto the descending axon of $c h \times 10^{+}$RSNs (Figure $3 G$ ).

\section{CSF-CN axons innervate the neuropil region in the caudal hindbrain}

The hindbrain houses a variety of neuronal populations expressing distinct neurotransmitters organized in stripes [79]. We inspected the axonal projections of CSFcNs relative to specific hindbrain neurons using specific transgenic lines labeling either glutamatergic (Tg(vglut2a:loxP-DsRed-loxP-GFP) [80]), GABAergic (Tg(gad1b:GFP) [81]), glycinergic (Tg(glyt2:GFP) [48]), or monoaminergic cells (Tg(vmat2:GFP) [82]) (Figure 4). In the caudal hindbrain, no obvious varicosities were seen between CSF-cNs 
and dorsally-located neuronal somata (Figure 4B-4E). However, we observed that CSF$\mathrm{cN}$ axons form a thin reticular lamina between dorsalmost somata and ventral neuropil region (Figure 4B-4E, dashed lines), where CSF-cN axons may form axo-axonic connections with the axons of dorsally-located neurons $\left(\mathrm{VMAT2}^{+}\right.$monoaminergic neurons shown in Video S5). We observed that CSF-cN axons do not synapse directly onto the somata of the inferior olive [83] but reach dorsally to it (Figure 4E3).

\section{Rostral CSF-cNs enhance tail bending amplitude and speed during the escape}

To investigate the behavioral contribution of rostralmost CSF-CNs projecting to caudal hindbrain, we ablated 60-70 CSF-cNs (Segments 4-9) using a 2-photon laser in $4 \mathrm{dpf}$ Tg(pkd211:GAL4;UAS:GFP) larvae (see STAR Methods). At $6 \mathrm{dpf}$, we compared the behavior of these larvae to a sham control group as well as to animals in which the same number of CSF-cNs were ablated in the caudalmost spinal cord (Segments 24-30) (Figure 5A). Larval zebrafish were tracked and kinematics were analyzed (Figure 5B, 5C) using an improved version of our open-source software ZebraZoom $[26,30,84]$ (https://zebrazoom.org/).

First, we verified that the ablation protocol did not lead to off-target effects, in particular on the RSN axons in close vicinity to CSF-cN somas. By performing 2-photon ablation of CSF-cNs in the triple transgenic Tg(pkd2/1:GAL4;UAS:GFP;To/056:GFP) larvae, in which both CSF-cNs and the Mauthner cell axons are $\mathrm{GFP}^{+}$, we confirmed that the ablation protocol spared the axons of the Mauthner cells in direct vicinity to the CSF-cN somas (Figure S2). We then used acousto-vestibular stimulus (AV) to induce short latency escape responses (Figure 5B2, S3A, S3B). The escape responses kinematics (Figure 5C3, 939 escapes from $n=103$ larvae) revealed no difference between caudally-ablated and control group (Figure 5D-5G). In contrast, compared to control siblings, larval zebrafish lacking rostralmost CSF-cNs exhibited escape response after a larger latency (Figure 5D, 22\% increase, Control: $5.0 \pm 0.2 \mathrm{~ms}$; Rostral: $6.1 \pm 0.2 \mathrm{~ms}, P=0.0016$ ), showed a C-bend with a smaller amplitude (Figure 5E, 16\% decrease: Control: $88.9 \pm$ 1.8 deg; Rostral: $74.9 \pm 2.2 \mathrm{deg}, P=1.1 \mathrm{e}-6$ ) and a larger time-to-peak (Figure $\mathbf{5 F}, \mathbf{6 \%}$ increase: Control: $8.1 \pm 0.1 \mathrm{~ms}$; Rostral: $8.6 \pm 0.1 \mathrm{~ms}, P=0.0189$ ). The reduced amplitude and delayed timing of the C-bend was associated with a $13 \%$ reduction in bout speed 
(Figure 5G, Control: $55.7 \pm 1.2 \mathrm{~mm} / \mathrm{s}$; Rostral: $48.2 \pm 1.1 \mathrm{~mm} / \mathrm{s}, P=4.4 \mathrm{e}-5$ ). The probability of a C-start was also decreased in rostral ablated fish (Figure $\mathbf{5 H}, \mathbf{2 3 . 1 \%}$ decrease in the C-start probability: Control: $0.9 \pm 0.01$; Rostral: $0.7 \pm 0.03$; Caudal: $0.9 \pm$ 0.01). Other kinematic parameters were similar among the three groups (Figure S3ES3L, Figure S4). In contrast, the ablation of rostralmost CSF-cNs had no effect on exploratory locomotion (Figure S5). The bout rate and kinematics of routine turns and forward bouts $[84,85]$ were similar for the three groups (Figure S5). These results reveal that rostralmost CSF-cNs specifically modulate the AV escape response.

\section{Rostralmost CSF-cNs contribute to active postural correction following the C-bend}

Previous work showed that the genetic blockade of neurotransmitter release in CSF-cNs increased the occurrence of rolling during AV escapes [39]. Subsets of RSNs contribute to initiation, steering as well as postural control together with cranial motor neurons $[20,67,86-88]$, we hypothesized that rostralmost CSF-cNs may control active posture via projections onto hindbrain targets.

To get an automated measurement of the postural defects over time, we developed a deep learning classifier that could estimate frame by frame the probability of occurrence of rolling posture (Figure 6A, Video S6). Based on the rolling probability over time during an escape (Figure 6B1), we defined a rolling event when the rolling probability exceeded $80 \%$. Then, we classified the rolling events as short and long rolling using a cutoff of 10 ms (Figure 6B1) and further build a classification of escape responses with three levels of postural defects (Figure 6B2): no rolling (no rolling events), moderate rolling (short rolling events only) and severe rolling (with at least one long rolling event). We then compared the rolling events between the three groups ( $n=103$ larvae, 939 escapes) and found no difference between the control and caudally-ablated group (Figure 6C1, $P=$ 0.6344; Figure 6C2, $P=0.9815)$. In contrast, larvae lacking rostralmost CSF-cNs showed more often postural defects shown as longer rolling duration (Figure 6C1, 59.5\% increase, Control: $12.6 \pm 1.3 \mathrm{~ms}$; Rostral: $20.1 \pm 1.8 \mathrm{~ms}, P=0.0181$ ) and an increase of total long rolling events (Figure 6C2, 59.5\% increase, Control: $0.42 \pm 0.05$; Rostral: 0.67 $\pm 0.06, P=0.0028$ ). Further investigation revealed that the difference among the three groups was only detected in the first five trials (Figure 6D1, 85.4\% increase in rolling 
duration, Control: $13.7 \pm 1.6 \mathrm{~ms}$; Rostral: $25.4 \pm 2.7 \mathrm{~ms}, P=0.0021$; Figure 6D2, 93.3\% increase in number of long rolling events, Control: $0.45 \pm 0.06$; Rostral: $0.87 \pm 0.09, P=$ $0.0005, \mathrm{n}=103$ larvae, 470 escapes).

To establish the onset of postural defects, we compared the rolling probability as a function of time for escape responses showing either no rolling (Figure 6E1; 40 for control, 28 for rostral and 28 for caudally-ablated group), moderate rolling (Figure 6E2; 61 for control, 89 for rostral and 26 for caudally-ablated group) or severe rolling (Figure 6E3; 91 for control, 74 for rostral and 33 for caudally-ablated group). In all escapes where a severe rolling occurred, the highest mean probability for the rolling events was observed between the counter bend and the third bend (gray box in Figure 6E3), which was consistent in all three groups (Figure S6). Our results indicate that rostralmost CSF-cNs contribute to active postural control during AV escapes by stabilizing balance after the powerful C-bend. Remarkably, pectoral fins have been shown dispensable for routine swimming in the roll and yaw control $[20,88]$. To test if pectoral fins are involved in the kinematics and posture control during escape responses, we mechanically ablated the pair of fins at $4 \mathrm{dpf}$ and analyzed the swimming kinematics and postural defects to AV stimuli at $6 \mathrm{dpf}$. We found no difference between the control and fins-ablated group (Figure S7), indicating that pectoral fins do not contribute to postural control in AV escape response.

\section{DISCUSSION}

We previously showed that CSF-cNs are sensory neurons detecting together with the Reissner fiber spinal curvature in an asymmetric manner $[30,34,35]$. While dorsolateral CSF-CNs detect spine bending to the left and right [30], ventral CSF-cNs detect longitudinal contractions [39]. Since CSF-cNs project one to six segments ascending and ipsilateral, rostralmost CSF-cNs in the spinal cord, whose soma is located in segments 4 - 9, project their axons into the hindbrain [46]. We investigated here the specific projections and functions carried out by these spinal sensory neurons to shape the kinematics of locomotion and active posture during swimming. We used a quantitative and high-throughput analysis of kinematic parameters as well as deep learning to dynamically estimate posture during active locomotion over thousands of escape 
responses. Using this automated approach, we show that during fast escapes, rostralmost CSF-cNs contribute first to boosting the locomotor speed by increasing the amplitude and shortening the timing of the initial C-bend, and second, after the C-bend, to stabilize posture. A fine anatomical and functional characterization reveals that these CSF-cNs in the rostral spinal cord massively synapse onto the descending axons of reticulospinal neurons involved in fast swimming and postural control, as well as onto MNs controlling the ventral musculatures of the head. By revealing that spinal interoceptive sensory neurons provide synaptic inhibition to the output of descending command neurons, our study provides novel insights on the circuit mechanisms underlying sensorimotor integration between spinal cord and hindbrain. The discovery of a direct inhibition of descending commands explains our previous observation that the optogenetic activation of the rostral, but not caudal CSF-cNs, disrupted the anteroposterior propagation of motor activity in the spinal cord [44]. Together, our body of work highlights a pivotal role of CSF-cNs in the rostrocaudal propagation of motor activity from the hindbrain down to the spinal cord during fast locomotion.

\section{Mechanosensory feedback via CSF-cNs targets the output of descending command neurons from hindbrain to spinal cord}

Sensory inputs from trigeminal, auditory and proprioceptive pathways modulate the activity of RSNs in the hindbrain via polysynaptic pathways involving intermediate nuclei $[7,8]$ or through the cerebellum $[89,90]$. However, up to date, no sensory neurons have been reported to directly project on descending command neurons. Here, we combine anatomical and molecular evidence using pre- and post-synaptic markers to show that CSF-cN mechanosensory feedback directly targets RSNs in larval zebrafish. CSF-cNs in the spinal cord form GABAergic axo-axonic synapses with the hindbrain command neurons as shown with the Mauthner cell as well as $\mathrm{Ch} \times 10^{+} \mathrm{RSNs}$ that are critical to shape locomotion across vertebrates [16,69,91-93]. Such direct targeting of sensory inputs onto descending command neurons has not been observed previously for trigeminal, auditory and proprioceptive sensory pathways. As shown by the juxtaposition of presynaptic markers from CSF-cNs and the presynaptic boutons of the Mauthner cell, the mechanisms of modulation of CSF-cNs onto the descending axons of the RSNs may involve presynaptic inhibition that could impact action potential propagation by modulation 
of synaptic release. The physiological mechanisms of RSN modulation by CSF-cNs will be the topic of another study.

GABAergic axo-axonic synapses are ubiquitous in the central nervous system. In the spinal cord, presynaptic inhibition corresponding to GABAergic axo-axonic synapses modulates the effects of primary afferents onto their spinal targets, which is associated with $G_{A B A_{A}}$ receptor mediated primary afferent depolarization (PAD) [94-97]. Such GABAergic presynaptic modulation has been also described since in the hippocampus and basal ganglia [98-100]. A recent investigation revealed a very similar profile of innervation for a subset of glycinergic inhibitory neurons only targeting axons of MNs in the spinal cord [101].

Interestingly, anatomical evidence in mammals indicate that CSF-cN projections are extensive in the ventral fissure [102], precisely where descending reticulospinal axons from the pons and vestibulospinal neurons are running as well [103], suggesting that axoaxonic projections may well be a conserved mechanism of CSF-cN modulation onto RSNs across vertebrate species.

\section{CSF-cN dependent circuit mechanisms to modulate locomotor speed and posture}

Our study reveals that rostralmost CSF-cNs contribute to the power and speed of escape responses as well as to the active control of posture after the C-start. We find that ventral CSF-cNs synapse in the hindbrain onto the dendrites and somas of occipital MNs projecting to the hypobranchial region, in a similar manner than the targeted projections onto $\mathrm{CaP}$ MNs in the spinal cord. In addition, we uncover a very different and specific map of connectivity onto Mauthner cell and $\mathrm{Ch} \times 10^{+} \mathrm{RSNs}$. While ventral CSF-cNs project onto Mauthner cell and early-born RSNs involved in fast and powerful movements, dorsolateral CSF-cNs project onto late-born RSNs involved in slow and refined movements. We can briefly speculate on the relation of such precise connectivity in regards to the control of locomotion and posture. During the oscillatory waves that propagate from rostral to caudal during swimming, the mechanosensory feedback from CSF-cNs constitute an efference copy that projects back and ipsilaterally to neurons in segments that were recently recruited. 
The very first movement of the AV escape is the rotation of the head away from the stimulus that is followed by the coordinated contraction of muscles along the tail to form the C-start $[104,105]$. The head positioning at the beginning of the C-start response is assumed to play a critical role in the accomplishment of the escape [104]. Our study demonstrates that CSF-cNs synapse onto occipital MNs controlling the head positioning. Lateral rotation of the head is largely carried out by primary MNs in the occipital column in the hindbrain while the bending of the tail for a C-start is due to CaP MNs in the spinal cord. The unilateral activation of these ventrally-projecting primary MNs in the hindbrain and spinal cord leads to a unilateral bend away from the stimulus together with a torque, which will recruit the ventral CSF-cNs. Upon activation, CSF-cNs synapse onto the very same MNs that initiated the escape response, thereby providing a negative feedback to forbid their sustained activity that would lead to a delayed activation, a slower C-start and rolling on one side.

Mauthner cells and early-born Chx10+ RSNs involved in fast escapes [69] and turning behavior $[65,66]$ send axons through the medial longitudinal fasciculus (MLF) to innervate motor circuits in the spinal cord $[91,106]$. We show here that ventral CSF-cNs form inhibitory synapses on the presynaptic boutons of the Mauthner cell and other RSNs involved in fast and powerful movements and projecting in the MLF. When the initial Cstart has been triggered, the CSF-cN feedback to fast RSNs could rapidly stop the descending command in order to keep the command short and effective, to rapidly switch to the counter-bend on the other side. Disruption of this ipsilateral stop command leads to a compromised $\mathrm{C}$-bend with smaller amplitude and slower kinetics, indicating that the sensory feedback contributes to the very first motor command. Subsets of RSNs in lamprey were shown to contribute to postural control $[107,108]$, CSF-CNs may also contribute via specific projections onto subsets of RSNs in order to fine tune active posture during fast swimming.

The C-start is a 3D maneuver in which the fish can pitch up or down to set its trajectory in 3D [104]. The startle response is composed of successive elements, including a Cstart with optional pitch, fast swimming, then slow swimming, which are controlled by circuits that can have different habituation rates $[104,109,110]$. Many kinematic parameters of the escape habituate after repetitive stimulations (see [30] and Figure S4: 
speed, duration and distance). In animals lacking rostralmost CSF-cNs, we noticed an attenuation of postural defects across trials (Figure 6C). The pitch described by Nair et al., 2015 may be the first component to habituate over time. Without such pitch, the Cstartle becomes a 2D maneuver in which the postural control may be not as tightly regulated by rostralmost CSF-cNs. Future studies will investigate whether the pitch component habituates using a $3 \mathrm{D}$ recording system operating at higher spatial resolution [111].

A large C-bend recruits the dorsolateral CSF-cNs [30,35,38]. We showed here that dorsolateral CSF-cNs mostly project onto the axon of RSNs in the lateral fasciculus (Figure 3F), which are recruited for slow exploration and refined movements [69]. Together with our previous evidence that dorsolateral CSF-cNs synapse on V0-V interneuron $[44,46]$, we can speculate that dorsolateral CSF-cNs when recruited during the large amplitude C-bend block the recruitment of the slow swimming circuits. Such contraction-dependent inhibition of slow swimming circuits during fast swimming can contribute along with V1 interneurons [112] to maximize locomotor speed by avoiding the mixing of fast and slow modules, which has been previously shown to slow down the locomotor rhythm [113]. Overall, we discovered a direct sensory feedback onto RSNs can complement the indirect feedback from the spinocerebellar tract that carries an efference copy to the hindbrain.

We had previously shown that the bilateral optogenetic activation of CSF-cNs in the rostralmost segments of the spinal cord leads to the complete stop of locomotion [44]. Our novel anatomical evidence further supports that CSF-cNs control the propagation of rostrocaudal activity in the spinal cord via the axo-axonic projections onto RSNs in addition to the inhibition of V0-v interneurons. CSF-cNs detect local curvature [30,39]. During slow swimming, the spinal curvature is limited to the caudal tail [84]. Therefore, middle and caudal CSF-cNs, but not rostralmost CSF-cNs, should be recruited during slow swimming. Accordingly, we observed no effect on slow swimming when rostralmost CSF-cNs were ablated (Figure S5).

We had shown that CSF-cNs project onto the primary MNs (see CaP [39] and occipital MNs, as shown in this study) and premotor V0-v interneurons [44], or synapse onto the 
axons of descending command neurons to modulate the rostrocaudal propagation of neuronal activity in locomotor circuits. Consistent with the connectivity map we established in the spinal cord $[39,44]$, we find that the dorsolateral and ventral CSF-cNs differently project to the fast and slow locomotor circuits. Our study likely did not identify yet all CSF-cN recipient neurons in the hindbrain. We have preliminary evidence showing that CSF-CN axons project onto the processes of monoaminergic neurons in the neuropil region (Video S5) as well as to unknown targets dorsal to the inferior olive (Figure 4E). Future investigations will be necessary to establish a full connectivity map.

Altogether, our study reveals that spinal sensory neurons in contact with the cerebrospinal fluid accomplish key functions for locomotion and posture via their projections onto brain targets. We show that acute ablations of rostral CSF-cNs recapitulates the effects on kinematics of the escape response observed after silencing neurotransmission of CSFcNs throughout the spinal cord [30], which reduced the amplitude of the C-bend and locomotor speed. By combining classical and molecular anatomy together with electrophysiology, we provide evidence that CSF-cN inputs can alter the descending command from the brain to spinal cord, as well as the activity of hindbrain MNs controlling head position. Our study provides evidence that spinal mechanosensory feedback can directly modulate the descending command information in order to shape complex integrative reflexes involving the brain and spinal cord.

\section{Figures and Legends}

\section{Figure 1. CSF-cNs project onto occipital motor neurons}

(A) CSF-cNs innervate motor neurons of Oc/Pec column. (A1) Genetic lines and schematic of the brain regions of interest of 4-5 dpf larvae. (A2) Optical projection stack of sections imaged from lateral side relative to the caudal hindbrain. CSF-cN axons project onto ventral Oc/Pec column but not the vagal motor column (X). (A3) Coronal view of the caudal hindbrain: cross-section resliced from the optical stacks of A2 (see dashed lines) to illustrate the projection pattern of CSF-cN axons. (A4) Z projection stack of a few optical sections acquired from the dorsal side at the caudal hindbrain. Note how CSF-cN axons (tagRFP ${ }^{+}$, green) terminate at the beginning of rhombomere 8. (A5) Z projection stack of optical sections imaged at the ventral level in the caudal hindbrain. (A5', A5') 
single planes of regions denoted in $\mathbf{A 5}$ showing putative connections between CSF-cN axons and lateral MNs (arrows). Note the basket structure of extensive varicosities encircling the soma of a motor neuron (arrow). Also see single planes in Video S1. (B) CSF-cNs innervate the lateral dendrites of occipital motor neurons. (B1) Z projection stack of a few optical sections imaged at the ventral level in the caudal hindbrain. CSF$\mathrm{CN}$ axons form synaptic boutons onto somas and dendrites of MNs. Region in dashed square is zoomed in (B1') at single plane. Note in (B1') the basket structure of extensive varicosities encircling the lateral and large soma of MN (indicated by arrows). (B2) shows the putative connections between CSF-cN axons and MN dendrites (arrowheads). (B2') shows the same region of interest at single plane. Also see single planes in Video S2. (C) An example of an occipital MN contacted by CSF-cNs that innervates the hypobranchial musculature. (C1) Loading of rhodamine in a rostralmost $\mathrm{MN}$ of the $\mathrm{Oc} / \mathrm{Pec}$ motor column that receives GABAergic inputs from CSF-cNs. (C2) Axial muscles of ventral jaw ventrally visualized by ChR2-YFP expressed via the UAS/GAL4 expression system using the $\alpha$-actin promoter. (C2') Axons of the filled MN terminate onto the ventral jaw muscle. The hypobranchial muscle in dashed is called the sternohyoideus (sh). (C2"') Axons of the labelled occipital MN innervate the entire sh muscle. Scale bars are $50 \mu \mathrm{m}$ in (A2-A4, B1, C1-C2) and $20 \mu \mathrm{m}$ in (A5, A5', A5', B1', B2, B2', C2', C2'). On top of the scale bars the striped squares denote if the image displayed is a maximal projection from multiple planes (three lines) or a single plane (single line). Dash lines in A4, A5, B1 indicate the border between hindbrain and spinal cord. HB: hindbrain; SC: spinal cord; DV: dorsal-ventral axis; R-C: rostral-caudal axis; r4-r8: Rhombomeres 4-8; M1-M4: Myotomes 1-4; VII: the seventh cranial nerve / facial nerve; $\mathrm{X}$ : the tenth cranial nerve / vagus nerve; Oc/Pec: Occipital-Pectoral motor column.

\section{Figure 2. CSF-cNs form GABAergic synapses onto occipital motor neurons}

(A) Experimental paradigm. Targeted whole-cell recordings of $\mathrm{GFP}^{+}$motor neuron while activating ChR2-mCherry ${ }^{+}$CSF-cNs using brief $(5 \mathrm{~ms})$ pulses of blue light. AlexaFluor 647 is loaded in the recording pipette with the internal solution to visualize the cell. In the shaded box, the blue bar represents the light pulse, the black line represents the light 
induced single spike in CSF-CN [44], and the red curve represents the IPSC detected in recorded motor neurons. (B) CSF-CN axons and varicosities surround an anterior and large occipital $\mathrm{MN}$ in a $4 \mathrm{dpf} T g\left(p k d 2 / 1: G A L 4 ; U A S: C h R 2-m C h e r r y ; p a r g a^{\text {mn2Et:GFP) }}\right.$ transgenic larva. Region in dashed line is zoomed in (B1) to show the extensive innervation (arrows). (B1') Image acquired after the electrophysiological recording showing the morphology of the recorded motor neuron. Note the dendritic structures protruding towards the lateral margin of the hindbrain. (C) Spontaneous bursting activity of a MN recorded in current-clamp mode. Region in shaded box is zoomed in C1'. (D) Current-clamp recording of targeted $\mathrm{MN}$ showing action potential firing in response to increasing step current injection of 10pA. Note the traces shown here are selected recordings with a total current step of 40-50pA. (E) Voltage-clamp recording of targeted MN during optical stimulation (blue bars) of ChR2-mCherry ${ }^{+}$CSF-cNs using a single 5 ms-long light pulse (E1) and a train of $205 \mathrm{~ms}$-long light pulses at $20 \mathrm{~Hz}$ (E2). $10 \mu \mathrm{M}$ AP5 and $10 \mu \mathrm{M} \mathrm{CNQX}$ were bath-applied to respectively block NMDA and AMPA receptors. As the holding potential $\mathrm{Vm}$ was $-75 \mathrm{mV}$ and reversal potential for chloride $E_{\mathrm{Cl}}{ }^{-}$was -51 $\mathrm{mV}$, IPSCs appear as inward currents. (F) Summary data showing IPSC amplitudes following light stimulation. Each experiment (circle) is the average of ten trials without (Off) and with (On) light pulse (mean \pm SEM: amplitude of light-off current change, $3.38 \pm$ $1.71 \mathrm{pA}$; amplitude of light evoked IPSC, $39.02 \pm 2.07 \mathrm{pA} ; \mathrm{n}=5$ cells from 5 fish, Paired $t$ test, $P=0.0004$ ). (G) The light-evoked IPSC (black trace, averaged from 10 trials in gray traces) was blocked with $10 \mu \mathrm{M}$ bath application of gabazine (red trace, averaged from 10 trials in gray traces) ( $n=2$ cells from 2 fish). ( $\mathbf{H}-\mathbf{K}$ ) Distribution of IPSC delay (I; mean \pm SEM: $5.9 \pm 0.1 \mathrm{~ms}$ ), current amplitude (J; mean \pm SEM: $39.1 \pm 1.9 \mathrm{pA}), 10 \%-90 \%$ rise time (K; mean \pm SEM: $1.62 \pm 0.13 \mathrm{~ms}$ ), and decay time $T$ (L; mean \pm SEM: $15.1 \pm 0.92$ $\mathrm{ms}$ ) ( $\mathrm{n}=5$ cells from 5 fish). The blue bar in (I) indicates the $5 \mathrm{~ms}$-long light pulse. Scale bars, 20 $\mathrm{mm}$ in (B), (B1) and (B1').

Figure 3. CSF-cNs project onto descending axons of reticulospinal neurons.

(A1) Z projection stack of a few optical sections acquired from the dorsal side relative to the caudal hindbrain of 4-5 dpf Tg(pkd2/1:tagRFP;To/056:GFP) larvae. Note the 
localization of Mauthner cells (white asterisk) relative to ascending CSF-cN projections (tagRFP ${ }^{+}$, green, the anterior-most axon terminal indicated by white arrow). (A2) Crosssection resliced from optical $Z$ stacks imaged from the dorsal side in order to show CSF$\mathrm{CN}$ projections onto the $\mathrm{GFP}^{+}$Mauthner axons (M axon) in caudal hindbrain (A2', arrowhead) and spinal cord (A2", arrowheads). (A3-A6) Single plane optical sections form the region denoted in dash boxes highlighting contacts from CSF-cN axons onto Mauthner axons (arrowheads) in hindbrain (A3-A4) as in spinal cord (A5-A6). See also Video S3.

(B-C) Dorsal, but not ventral, CSF-cNs synapse onto descending axons of Mauthner cells in the MLF. CSF-cNs are sparsely-labeled by injection of construct (UAS:synaptophysin$m$ Cherry) in Tg(pkd2/1:GAL4;Tol056:GFP) eggs. (B1) A ventral CSF-cN labeled possess numerous synapses onto the $\mathrm{GFP}^{+}$descending axon of the Mauthner cell. The top inset represents a coronal view of the ventral and dorsal population of CSF-cNs present in the spinal cord. (B1', B1') Single plane of the regions denoted in dashed square in (B1) to show the presynaptic boutons of CSF-cN axon. (B2, B3) Ventral CSF-cNs form varicosities on close apposition to the boutons of Mauthner cell axons. Single CSF-CN is labeled by Synaptophysin-mCherry as in (B1). (B2) and (B3) show two examples of CSF$\mathrm{cN}$ varicosities in close contact with the boutons of Mauthner axon indicated by white arrowheads. (C) A dorsolateral CSF-cN rarely form synapses onto the descending axon of Mauthner cell. (C', C') Single plane of the regions denoted in dashed square in (C) to show the presynaptic boutons of CSF-cN axon in relation to the Mauthner axon. Note that at the single plane level no close proximity is found between the CSF-CN projection and the Mauthner axons. (D) CSF-cNs form varicosities on descending fibers of the V2a reticulospinal neurons in the hindbrain and spinal cord of 4-5 dpf Tg(pkd211:tagRFP;chx10:GFP) larvae. (E, F) CSF-cNs are sparsely-labeled by injection of construct of (UAS:Synaptophysin-mCherry) in Tg(pkd2/1:GAL4;chx10:GFP) eggs. (E) A ventral CSF-cN labeled projects onto V2a axons in MLF. (E') Single plane of the region denoted in dashed square in (E) to show the presynaptic boutons of CSF-CN axon in relation to the $\mathrm{GFP}^{+} \mathrm{V} 2 \mathrm{a}$ axon in MLF (contacts indicated by arrowheads). (F) A dorsolateral CSF-cN labeled. (F') Single plane of the region denoted in dashed square in (F) to show the labeled CSF-cN did not project to GFP+ V2a axons in MLF but formed 
synapses onto the V2a axons in the lateral fasciculus in the spinal cord (contacts indicated by arrowheads). (G) CSF-cN axons form inhibitory synapses onto descending axons of Chx $10^{+}$RSNs. Chx $10^{+} \mathrm{RSNs}$ are sparsely labeled by injection of construct (pT2MUAS:mCherry-zGephyrin-aP1) in Tg(pkd2/1:GFP;chx10:GAL4) eggs. (G1-G4) are zoomed from regions in (G) to show that the $\mathrm{GFP}^{+} \mathrm{CSF}-\mathrm{cN}$ varicosities are in close vicinity with $\mathrm{mCherry}^{+}$postsynaptic densities of the descending axon of $\mathrm{Ch} \times 10^{+} \mathrm{RSNs}$ (indicated by white arrow heads) in the neuropil region of caudal hindbrain. Scale bars are $50 \mu \mathrm{m}$ in (A1, D, G), $20 \mu \mathrm{m}$ in (A2-A6, C, E, F) and $10 \mu \mathrm{m}$ in (B1, B2, B3, G1-G4); Symbols as in Figure 1.

Figure 4. CSF-cNs project onto the caudal hindbrain neuropil region

(A) Schematic of the brain regions of interest of 4-5 dpf larvae. (B-E) Coronal view of CSF-cN projection patterns at caudal hindbrain in transgenic lines labeling different transmitter systems: GABAergic neurons (B, Tg(pkd211:GAL4;UAS:ChR2mCherry;gad1b:GFP)), glycinergic neurons (C, Tg(pkd2l1:GAL4;UAS:ChR2$m$ Cherry;glyt2:GFP)), monoaminergic neurons (D, Tg(pkd2/1:tagRFP;vmat2:GFP)) and glutamatergic neurons (E, Tg(pkd2/1:GAL4;UAS:ChR2-YFP;vglut2a:loxP-DsRed-IoxPGFP)). CSF-CN axons form a reticular lamina between the dorsal somata and ventral neuropil (indicated by dashed lines). Note that the CSF-cN reticular lamina is aligned with the axis of the monoaminergic column in (D). (E) CSF-cNs rarely contact VGluT2a+ glutamatergic neurons in the caudal hindbrain and do not project axons to the inferior olive. (E1-E2) Z-projection stack (depth $\sim 10 \mu \mathrm{m}$ ) of optical sections at different dorsalventral levels of caudal hindbrain revealed no connections between CSF-cNs and glutamatergic neurons. (E3) at the ventralmost level, the inferior olive (IO) receives no inputs from CSF-cNs. Scale bars are $20 \mu \mathrm{m}$ in (B-E) and $50 \mu \mathrm{m}$ in (E1-E3). Symbols as in Figure 1.

Figure 5. Hindbrain-projecting CSF-cNs modulate the power and locomotor speed during escapes 
(A) Ablations were performed on $4 \mathrm{dpf} T g$ (pkd2/1:GAL4;UAS:GFP) larvae using a 2photon laser (A1). Siblings anesthetized and embedded in agarose were used as control larvae ( $n=41$ larvae, $\mathbf{A 2}$, top) to be compared to larvae in which 60-70 cell ablations were performed onto either rostral CSF-cNs ( $n=43$ larvae) (A2') or caudal CSF-cNs ( $n=19$ larvae) (A2"). (B1) Larvae were treated with $4.5 \mu \mathrm{g} / \mathrm{mL}$ PTU from 0 to $4 \mathrm{dpf}$, when the cell ablation was performed, and then transferred to system water to recover before behavioral testing at $6 \mathrm{dpf}$. (B2) Prior to the behavioral experiment, fish were allowed to acclimate to the arena for $10 \mathrm{~min}$. A 5 min-long recording of exploration was followed by 10 acousto-vestibular (AV) stimuli (3 min inter-trial intervals) to induce escape responses. (C) Experimental setup built for AV stimuli on freely swimming larvae. (C1) AV stimuli were generated with two speakers. (C2) Superimposed images showing the trajectory of a typical AV escape response across time. (C3) Tail angle tracked over time to define the escape latency, C-bend amplitude and time to peak of the C-bend. Vertical grey line indicates the AV stimulus. Vertical dash line indicates the onset of the escape. Green circle indicates the C-bend peak amplitude. (D) Larvae deprived of rostralmost CSF-cNs showed an increased escape latency compared to control siblings (Wald $X^{2}(2)=12.94, P$ $=0.0016$; Tukey's post hoc analysis: $P=0.0016$ for Control versus Rostral, $P=0.1955$ for Caudal versus Rostral; $P=0.6346$ for Control versus Caudal). (E) Larvae deprived of rostralmost CSF-cNs showed a decreased C-bend amplitude compared to control siblings (Wald $X^{2}(2)=39.22, P=3.1 \mathrm{e}-9$; Tukey's post hoc analysis: $P=1.1 \mathrm{e}-6$ for Control versus Rostral, $P=1.6 \mathrm{e}-5$ for Caudal versus Rostral, $P=0.7257$ for Control versus Caudal). (F) Larvae deprived of rostralmost CSF-cNs showed an increased C-bend time-to-peak (Wald $X^{2}(2)=13.13, P=0.0014$; Tukey's post hoc analysis: $P=0.0189$ for Control versus Rostral, $P=0.0061$ for Caudal versus Rostral, $P=0.5125$ for Control versus Caudal). (G) Larvae deprived of rostralmost CSF-cNs showed a decreased bout speed (Wald $X^{2}(2)=$ 21.30, $P=2.4 \mathrm{e}-5$; Tukey's post hoc analysis: $P=4.4 \mathrm{e}-5$ for Control versus Rostral, $P=$ 0.0505 for Caudal versus Rostral, $P=0.5698$ for Control versus Caudal). (H) Larvae lacking rostral CSF-cNs executed less often the C-start that is characterized by an initial tail bend amplitude larger than 60 degrees $(23.1 \%$ decrease in the C-start probability: Control: $0.9352 \pm 0.0168$; Rostral: $0.7189 \pm 0.0385$; Caudal: $0.9528 \pm 0.0187$ ). KruskalWallis test, $X^{2}(2)=29.01, P<0.0001$. Post hoc, Dunn's multiple comparisons test. $P<$ 
0.0001 for Control versus Rostral, $P>0.9999$ for Control versus Caudal, $P=0.0002$ for Rostral versus Caudal. For all parameters: Control group: $n=41$ larvae from 5 clutches; Rostral CSF-cNs ablated group: $\mathrm{n}=43$ larvae from 5 clutches; Caudal CSF-cNs ablated group: $n=19$ larvae from 3 clutches. In the violin plots, each circle represents the mean value of up to 10 escape responses from each larva. The black line indicates the median value and the red line indicates the mean value for each group. Data are presented as mean \pm SEM. ANOVA, Type II Wald $X^{2}$ test. Post hoc analysis: Bonferroni correction, as 8 parameters have been analyzed together (see Figure S3A-S3H, Figure S4), the $P$ value from ANOVA test needs to be lower than $0.0063(0.05 / 8)$ to sign a significant difference. Turkey's multiple comparison test was used for the post hoc comparisons between each two groups.

Figure 6. Ablation of rostral CSF-cNs leads to postural defects during fast escape (A) Workflow for the automated rolling classification with the deep learning network. See STAR Methods.

(B) Zebrafish larvae showed different levels of postural defects during AV escape responses. (B1) Sample traces of rolling probability (Rp) estimated by the deep learning classifier showing escapes with no rolling, short rolling and long rolling events. Dashed line indicates the threshold $(R p=0.8)$ to detect a rolling event. Green bar indicates the short rolling event (duration $<10 \mathrm{~ms}$ ) while red bar indicates the long rolling event (duration > $10 \mathrm{~ms}$ ). (B2) Sequence of images from recorded videos showing escapes corresponding to (B1) with different levels of postural defects. The orange dots indicate the occurrence of rolling.

(C) Larvae lacking rostralmost CSF-cNs showed increased postural defects compared to control siblings. (C1) Rolling duration. Treatment effect: Wald $X^{2}(2)=12.37, P=0.0021$; Trial effect: Wald $X^{2}(1)=20.45, P=6.1 \mathrm{e}-6$; Tukey's post hoc analysis: $P=0.0181$ for Control versus Rostral, $P=0.6344$ for Control versus Caudal, $P=0.0105$ for Rostral versus Caudal. (C2) Number of long rolling events per escape. Treatment effect: Wald $X^{2}(2)=13.11, P=0.0014$; Trial effect: Wald $X^{2}(1)=21.57, P=3.4 \mathrm{e}-6$; Tukey's post hoc 
analysis: $P=0.0028$ for Control versus Rostral, $P=0.9815$ for Control versus Caudal, $P$ $=0.0207$ for Rostral versus Caudal.

(D) The significant difference between rostrally-ablated and control siblings was present over the first five out of the ten trials. ANOVA, Type II Wald $X^{2}$ test. Turkey's multiple comparison test was used for the post hoc comparisons between each two groups. (D1) Rolling duration. Wald $X^{2}(2)=15.16, P=5.1 \mathrm{e}-4$; Tukey's post hoc analysis: $P=0.0021$ for Control versus Rostral, $P=0.4436$ for Control versus Caudal, $P=7.1 \mathrm{e}-4$ for Rostral versus Caudal. (D2) Number of long rolling events per escape. Wald $X^{2}(2)=17.67, P=$ 1.5e-4; Tukey's post hoc analysis: $P=5.2 \mathrm{e}-4$ for Control versus Rostral, $P=0.9081$ for Control versus Caudal, $P=0.0040$ for Rostral versus Caudal. In the violin plot, each circle represents the mean value of up to 5 escape responses from each larva; black line indicates the median value and the red line indicates the mean value for each group. Data are presented as mean \pm SEM. Post hoc analysis: Bonferroni correction, the $P$ value from ANOVA test needs to be lower than $0.025(0.05 / 2)$ to sign a significant difference.

(E) Rolling events started mostly after the initial C-bend. For escapes with no rolling (E1), moderate rolling (E2), and severe rolling trials (E3) the mean tail angle over time is shown in dashed lines, the mean rolling probability in solid lines and the raster plot shows the rolling probability over time. Although all three groups showed rolling events, the rostrallyablated larvae showed more often long rolling events (46.6\%) compared to the caudallyablated (29.9\%) and control siblings (31.7\%). (E3) The onset of long rolling events was consistent among three groups (gray segment under rolling probability trace, see Figure S6).

\section{ACKNOWLEDGMENTS}

We thank Sophie Nunes, Monica Dicu and Antoine Arneau from Animalliance for fish care. We thank Prof. Philip W. Ingham (NTU, Singapore) for providing the plasmid of $\alpha-$ actin promoter in order for us to generate the $T g\left(\alpha\right.$-actin:GAL4) ${ }^{i c m 51}$ transgenic fish line. We thank the Riken Institute for sharing the Tg(gfap:GAL4) and $T g(z C R E S T$-hsp70:GFP) transgenic fish lines and Prof. Hiromi Hirata for kindly sharing the plasmid pT2MUAS:mCherry-zGephyrin-aP1. This work was supported by an ERC Starting Grants 
"Optoloco" 331 \#311673, New York Stem Cell Foundation (NYSCF) Robertson Award 2016 Grant 332 \#NYSCF-R-NI39, the HFSP Program Grants \#RGP0063/2014 and \#RGP0063/2018, the NIH Grant \#1U19NS104653-01. M.C-T. was supported by Campus France PRESTIGE postdoctoral research fellowship \#2017-2-0035. M-Y.W. received his doctoral fellowship from École des Neurosciences de Paris Île-de-France (ENP).

\section{AUTHOR CONTRIBUTIONS}

M-Y.W. performed cell ablation, in vivo electrophysiological recordings and behavior assays. M-Y.W. performed imaging experiments with the help of M.C-T.; M-Y.W. and O.M. ran behavior analysis and developed together the deep learning algorithm; F.Q. generated custom scripts to analyze the kinematics of movement; J.R generated the transgenic lines and performed the plasmid injection at single cell stage; F-X.L. helped with the behavioral data statistics in R; K.F did the initial anatomical observations. M-Y.W. and C.W. together designed experiments, analyzed data; C.W. conceived and supervised the project; M-Y.W., M.C-T. and C.W. wrote the manuscript with the inputs of all authors.

\section{DECLARATION OF INTERESTS}

The authors declare no conflict of interest.

\section{REFERENCES}

1. Kiehn, O. (2006). Locomotor Circuits in the Mammalian Spinal Cord. Annu. Rev. Neurosci. 29, $279-306$.

2. Grillner, S., and El Manira, A. (2015). The intrinsic operation of the networks that make us locomote. Curr. Opin. Neurobiol., 31:244-249.

3. Grillner, S., and El Manira, A. (2020). Current Principles of Motor Control, with Special Reference to Vertebrate Locomotion. Physiol. Rev. 100, 271-320.

4. Grillner, S., Wallén, P., Saitoh, K., Kozlov, A., Robertson, B., Wallen, P., Saitoh, K., Kozlov, A., and Robertson, B. (2008). Neural bases of goal-directed locomotion in vertebrates--an overview. Brain Res Rev 57, 2-12.

5. Di Prisco, G.V., Ohta, Y., Bongianni, F., Grillner, S., and Dubuc, R. (1995). Trigeminal inputs to reticulospinal neurones in lampreys are mediated by excitatory and inhibitory amino acids. Brain Res.

6. Korn, H., and Faber, D.S. (2005). The Mauthner cell half a century later: A neurobiological model for decision-making? Neuron 47, 13-28.

7. Daghfous, G., Green, W.W., Alford, S.T., Zielinski, B.S., and Dubuc, R. (2016). Sensory activation of command cells for locomotion and modulatory mechanisms: Lessons from lampreys. Front. Neural Circuits, 10:18.

8. Di Prisco, G.V., Boutin, T., Petropoulos, D., Brocard, F., and Dubuc, R. (2005). The trigeminal sensory relay to reticulospinal neurones in lampreys. Neuroscience $131,535-546$.

9. Dubuc, R., Brocard, F., Antri, M., Fénelon, K., Gariépy, J.F., Smetana, R., Ménard, A., Le Ray, D., Viana Di Prisco, G., Pearlstein, É., et al. (2008). Initiation of locomotion in lampreys. Brain Res. Rev. 57, 172-182. 
10. Jordan, L.M., Liu, J., Hedlund, P.B., Akay, T., and Pearson, K.G. (2008). Descending command systems for the initiation of locomotion in mammals. Brain Res. Rev. 57, 183-191.

11. Brocard, F., and Dubuc, R. (2003). Differential contribution of reticulospinal cells to the control of locomotion induced by the mesencephalic locomotor region. J. Neurophysiol. 90, 1714-1727.

12. Drew, T., Dubuc, R., and Rossignol, S. (1986). Discharge patterns of reticulospinal and other reticular neurons in chronic, unrestrained cats walking on a treadmill. J. Neurophysiol. 55, 375-401.

13. Deliagina, T.G., Zelenin, P. V., Fagerstedt, P., Grillner, S., and Orlovsky, G.N. (2000). Activity of reticulospinal neurons during locomotion in the freely behaving lamprey. J. Neurophysiol. 83, 853-63.

14. Perrins, R., Walford, A., and Roberts, A. (2002). Sensory Activation and Role of Inhibitory Reticulospinal Neurons that Stop Swimming in Hatchling Frog Tadpoles. J. Neurosci. 22, 4229-4240.

15. Juvin, L., Grätsch, S., Trillaud-Doppia, E., Gariépy, J.F., Büschges, A., and Dubuc, R. (2016). A Specific Population of Reticulospinal Neurons Controls the Termination of Locomotion. Cell Rep. 15, 2377-2386.

16. Bouvier, J., Caggiano, V., Leiras, R., Caldeira, V., Bellardita, C., Balueva, K., Fuchs, A., and Kiehn, O. (2015). Descending Command Neurons in the Brainstem that Halt Locomotion. Cell 163, 1191-1203.

17. Aksay, E., Gamkrelidze, G., Seung, H.S., Baker, R., and Tank, D.W. (2001). In vivo intracellular recording and perturbation of persistent activity in a neural integrator. Nat. Neurosci. 4, 184-193.

18. Clark, C., Austen, O., Poparic, I., and Guthrie, S. (2013). A2-Chimaerin Regulates a Key Axon Guidance Transition During Development of the Oculomotor Projection. J. Neurosci. 33, 16540-16551.

19. Green, M.H., and Hale, M.E. (2012). Activity of pectoral fin motoneurons during two swimming gaits in the larval zebrafish (Danio rerio) and localization of upstream circuit elements. J. Neurophysiol. 108, 3393-3402.

20. Green, M.H., Ho, R.K., and Hale, M.E. (2011). Movement and function of the pectoral fins of the larval zebrafish (Danio rerio) during slow swimming. J. Exp. Biol. 214, 3111-3123.

21. Higashijima, S., Hotta, Y., and Okamoto, H. (2000). Visualization of cranial motor neurons in live transgenic zebrafish expressing green fluorescent protein under the control of the islet-1 promoter/enhancer. J. Neurosci. 20, 206-18.

22. Ma, L.H., Gilland, E., Bass, A.H., and Baker, R. (2010). Ancestry of motor innervation to pectoral fin and forelimb. Nat. Commun. 1, 1-8.

23. Thorsen, D.H., and Hale, M.E. (2007). Neural development of the zebrafish (Danio rerio) pectoral fin. J. Comp. Neurol.

24. Uemura, Y., Kato, K., Kawakami, K., Kimura, Y., Oda, Y., and Higashijima, S.I. (2020). Neuronal Circuits That Control Rhythmic Pectoral Fin Movements in Zebrafish. J. Neurosci. 40, 6678-6690.

25. Buchanan, J.T. (2018). Swimming rhythm generation in the caudal hindbrain of the lamprey. J. Neurophysiol. 119, 1681-1692.

26. Knafo, S., Fidelin, K., Prendergast, A., Tseng, P.B.E.B., Parrin, A., Dickey, C., Böhm, U.L., Figueiredo, S.N., Thouvenin, O., Pascal-Moussellard, H., et al. (2017). Mechanosensory neurons control the timing of spinal microcircuit selection during locomotion. Elife 6.

27. Rossignol, S., Dubuc, R., and Gossard, J.P. (2006). Dynamic sensorimotor interactions in locomotion. Physiol. Rev. 86, 89-154.

28. Akay, T., Tourtellotte, W.G., Arber, S., and Jessell, T.M. (2014). Degradation of mouse locomotor pattern in the absence of proprioceptive sensory feedback. Proc. Natl. Acad. Sci. U. S. A. 111, 16877-16882.

29. Tuthill, J.C., and Azim, E. (2018). Proprioception. Curr. Biol. 28, R194-R203.

30. Böhm, U.L., Prendergast, A., Djenoune, L., Figueiredo, S.N., Gomez, J., Stokes, C., Kaiser, S., Suster, M., Kawakami, K., Charpentier, M., et al. (2016). CSF-contacting neurons regulate locomotion by relaying mechanical stimuli to spinal circuits. Nat. Commun. 7, 1-8.

31. Kolmer, W. (1921). Das „Sagittalorgan“ der Wirbeltiere. Z. Anat. Entwicklungsgesch. 60, 652-717.

32. Agduhr, E. (1922). Über ein zentrales Sinnesorgan (?) bei den Vertebraten. Z. Anat. Entwicklungsgesch. 66, 223-360. 
33. Wyart, C., Del Bene, F., Warp, E., Scott, E.K., Trauner, D., Baier, H., and Isacoff, E.Y. (2009). Optogenetic dissection of a behavioural module in the vertebrate spinal cord. Nature 461, 407-410.

34. Sternberg, J.R., Prendergast, A.E., Brosse, L., Cantaut-Belarif, Y., Thouvenin, O., Orts-Del'Immagine, A., Castillo, L., Djenoune, L., Kurisu, S., McDearmid, J.R., et al. (2018). Pkd2I1 is required for mechanoception in cerebrospinal fluid-contacting neurons and maintenance of spine curvature. Nat. Commun. 9, 1-10.

35. Orts-Del'Immagine, A., Cantaut-Belarif, Y., Thouvenin, O., Roussel, J., Baskaran, A., Langui, D., Koëth, F., Bivas, P., Lejeune, F.-X., Bardet, P.-L., et al. (2020). Sensory neurons contacting the cerebrospinal fluid require the Reissner fiber to detect spinal stretch in vivo. Curr. Biol., In press.

36. Jalalvand, E., Robertson, B., Wallen, P., Grillner, S., Wallén, P., and Grillner, S. (2016). Ciliated neurons lining the central canal sense both fluid movement and pH through ASIC3. Nat Commun 7, 10002.

37. Vigh, B., and Vigh-Teichmann, I. (1998). Actual problems of the cerebrospinal fluid-contacting neurons. Microsc. Res. Tech., 41:57-83.

38. Desban, L., Prendergast, A., Roussel, J., Rosello, M., Geny, D., Wyart, C., and Bardet, P.L. (2019). Regulation of the apical extension morphogenesis tunes the mechanosensory response of microvilliated neurons. PLoS Biol. 17(4): e30.

39. Hubbard, J.M., Bohm, U.L., Prendergast, A., Tseng, P.E.B., Newman, M., Stokes, C., and Wyart, C. (2016). Intraspinal Sensory Neurons Provide Powerful Inhibition to Motor Circuits Ensuring Postural Control during Locomotion. Curr Biol 26, 2841-2853.

40. Rose, C., Pompili, D., Henke, K., Van Gennip, J., Meyer-Miner, A., Rana, R., Gobron, S., Harris, M., Nitz, M., and Ciruna, B. (2019). SCO-Spondin Defects and Neuroinflammation Identified as Conserved Mechanisms Driving Severe Spine Deformity Across Genetic Models of Idiopathic Scoliosis. SSRN Electron. J.

41. Troutwine, B., Gontarz, P., Minowa, R., Monstad-rios, A., Mia, J., Sepich, D.S., Kwon, R.Y., Solnica-krezel, L., and Ryan, S. (2019). The Reissner Fiber is Highly Dynamic in vivo and Controls Morphogenesis of the Spine. bioRxiv preprint.

42. Zhang, X., Jia, S., Chen, Z., Chong, Y.L., Xie, H., Feng, D., Wu, X., Song, D.Z., Roy, S., and Zhao, C. (2018) Cilia-driven cerebrospinal fluid flow directs expression of urotensin neuropeptides to straighten the vertebrate body axis. Nat. Genet. 50, 1666-1673.

43. Cantaut-Belarif, Y., Sternberg, J.R., Thouvenin, O., Wyart, C., and Bardet, P.L. (2018). The Reissner Fiber in the Cerebrospinal Fluid Controls Morphogenesis of the Body Axis. Curr. Biol. 28, 2479-2486.e4.

44. Fidelin, K., Djenoune, L., Stokes, C., Prendergast, A., Gomez, J., Baradel, A., Del Bene, F., and Wyart, C. (2015). State-dependent modulation of locomotion by GABAergic spinal sensory neurons. Curr. Biol. 25, 3035-3047.

45. Djenoune, L., Khabou, H., Joubert, F., Quan, F.B., Nunes Figueiredo, S., Bodineau, L., Del Bene, F., Burckle, C., Tostivint, H., and Wyart, C. (2014). Investigation of spinal cerebrospinal fluid-contacting neurons expressing PKD2L1: evidence for a conserved system from fish to primates. Front Neuroanat 8, 26.

46. Djenoune, L., Desban, L., Gomez, J., Sternberg, J.R., Prendergast, A., Langui, D., Quan, F.B., Marnas, H., Auer, T.O., Rio, J.P., et al. (2017). The dual developmental origin of spinal cerebrospinal fluid-contacting neurons gives rise to distinct functional subtypes. Sci Rep 7, 719.

47. Ritter, D.A., Bhatt, D.H., and Fetcho, J.R. (2001). In vivo imaging of zebrafish reveals differences in the spinal networks for escape and swimming movements. J Neurosci 21, 8956-8965.

48. McLean, D.L., Fan, J., Higashijima, S.I., Hale, M.E., and Fetcho, J.R. (2007). A topographic map of recruitment in spinal cord. Nature $446,71-75$.

49. McLean, D.L., Masino, M.A., Koh, I.Y., Lindquist, W.B., and Fetcho, J.R. (2008). Continuous shifts in the active set of spinal interneurons during changes in locomotor speed. Nat Neurosci 11, 1419-1429.

50. Björnfors, E.R., El Manira, A., Bjornfors, E.R., and El Manira, A. (2016). Functional diversity of excitatory commissural interneurons in adult zebrafish. Elife 5, 1-15.

51. Myers, P.Z., Eisen, J.S., and Westerfield, M. (1986). Development and axonal outgrowth of identified motoneurons in the zebrafish. J. Neurosci. 6, 2278-2289. 
52. Bagnall, M.W., and McLean, D.L. (2014). Modular organization of axial microcircuits in zebrafish. Science (80-. ). 343, 197-200.

53. Knogler, L.D., and Drapeau, P. (2014). Sensory gating of an embryonic zebrafish interneuron during spontaneous motor behaviors. Front Neural Circuits 8, 121.

54. Higashijima, S., Schaefer, M., Fetcho, J. R. (2004). Neurotransmitter properties of spinal interneurons in embryonic and larval zebrafish. J Comp Neurol 480(1), 19-37.

55. Pflieger, J.F., and Dubuc, R. (2004). Vestibulo-reticular projections in adult lamprey: Their role in locomotion. Neuroscience 129, 817-829.

56. Kohashi, T., and Oda, Y. (2008). Initiation of Mauthner- or non-Mauthner-mediated fast escape evoked by different modes of sensory input. J Neurosci 28, 10641-10653.

57. Takakusaki, K. (2017). Functional Neuroanatomy for Posture and Gait Control. J. Mov. Disord. 10, 1-17.

58. Thorsen, D.H., and Hale, M.E. (2005). Development of zebrafish (Danio rerio) pectoral fin musculature. J. Morphol. 266, 241-255.

59. Balciunas, D., Davidson, A.E., Sivasubbu, S., Hermanson, S.B., Welle, Z., and Ekker, S.C. (2004). Enhancer trapping in zebrafish using the Sleeping Beauty transposon. BMC Genomics 5, 62.

60. Uemura, O., Okada, Y., Ando, H., Guedj, M., Higashijima, S.I., Shimazaki, T., Chino, N., Okano, H., and Okamoto, H. (2005). Comparative functional genomics revealed conservation and diversification of three enhancers of the isl1 gene for motor and sensory neuron-specific expression. Dev. Biol. 278, 587-606.

61. Myers, P.Z. (1985). Spinal motoneurons of the larval zebrafish. J. Comp. Neurol. 236, 555-561.

62. Diogo, R., Hinits, Y., and Hughes, S.M. (2008). Development of mandibular, hyoid and hypobranchial muscles in the zebrafish: homologies and evolution of these muscles within bony fishes and tetrapods. BMC Dev. Biol. 8:24.

63. Issa, F.A., Mazzochi, C., Mock, A.F., and Papazian, D.M. (2011). Spinocerebellar Ataxia Type 13 Mutant Potassium Channel Alters Neuronal Excitability and Causes Locomotor Deficits in Zebrafish. J. Neurosci. 31, 6831-6841.

64. Severi, K.E., Portugues, R., Marques, J.C., O’Malley, D.M., Orger, M.B., Engert, F., Malley, D.M.O., Orger, M.B., and Engert, F. (2014). Neural Control and Modulation of Swimming Speed in the Larval Zebrafish. Neuron 83, 692-707.

65. Orger, M.B., Kampff, A.R., Severi, K.E., Bollmann, J.H., and Engert, F. (2008). Control of visually guided behavior by distinct populations of spinal projection neurons. Nat. Neurosci. 11, 327-333.

66. Huang, K.H., Ahrens, M.B., Dunn, T.W., and Engert, F. (2013). Spinal projection neurons control turning behaviors in zebrafish. Curr Biol 23, 1566-1573.

67. Thiele, T.R., Donovan, J.C., and Baier, H. (2014). Descending control of swim posture by a midbrain nucleus in zebrafish. Neuron 83, 679-691.

68. Bhattacharyya, K., McLean, D.L., and Maclver, M.A. (2017). Visual Threat Assessment and Reticulospinal Encoding of Calibrated Responses in Larval Zebrafish. Curr. Biol. 27, 2751-2762.e6.

69. Pujala, A., and Koyama, M. (2019). Chronology-based architecture of descending circuits that underlie the development of locomotor repertoire after birth. Elife 8, e42135.

70. Liu, K.S., and Fetcho, J.R. (1999). Laser ablations reveal functional relationships of segmental hindbrain neurons in zebrafish. Neuron 23, 325-335.

71. Kohashi, T., Nakata, N., and Oda, Y. (2012). Effective sensory modality activating an escape triggering neuron switches during early development in zebrafish. J Neurosci 32, 5810-5820.

72. Takahashi, M., Inoue, M., Tanimoto, M., Kohashi, T., and Oda, Y. (2017). Short-term desensitization of fast escape behavior associated with suppression of Mauthner cell activity in larval zebrafish. Neurosci. Res. 121, 29-36.

73. Eaton, R.C., Lee, R.K.K., and Foreman, M.B. (2001). The Mauthner cell and other identified neurons of the brainstem escape network of fish. Prog. Neurobiol. 63, 467-485. 
Wu et al. revised manuscript to Current Biology

74. Sillar, K.T. (2009). Quick guide Mauthner cells. Curr. Biol. 19, R353-355.

75. Funch, P.G., and Faber, D.S. (1982). Cable properties of goldfish Mauthner axon. J. Neurophysiol. 47, 11961213.

76. Satou, C., Kimura, Y., Kohashi, T., Horikawa, K., Takeda, H., Oda, Y., and Higashijima, S. (2009). Functional role of a specialized class of spinal commissural inhibitory neurons during fast escapes in zebrafish. J Neurosci 29, 6780-6793.

77. Kimura, Y., Okamura, Y., and Higashijima, S. (2006). alx, a zebrafish homolog of Chx10, marks ipsilateral descending excitatory interneurons that participate in the regulation of spinal locomotor circuits. $\mathrm{J}$ Neurosci 26, 5684-5697.

78. Ogino, K., Yamada, K., Nishioka, T., Oda, Y., Kaibuchi, K., and Hirata, H. (2019). Phosphorylation of Gephyrin in Zebrafish Mauthner Cells Governs Glycine Receptor Clustering and Behavioral Desensitization to Sound. J. Neurosci. 39, 8988-8997.

79. Kinkhabwala, A., Riley, M., Koyama, M., Monen, J., Satou, C., Kimura, Y., Higashijima, S. -i., and Fetcho, J. (2011). A structural and functional ground plan for neurons in the hindbrain of zebrafish. Proc. Natl. Acad. Sci. 108, 1164-1169.

80. Koyama, M., Kinkhabwala, A., Satou, C., Higashijima, S., and Fetcho, J. (2011). Mapping a sensory-motor network onto a structural and functional ground plan in the hindbrain. Proc Natl Acad Sci U S A 108, 11701175.

81. Satou, C., Kimura, Y., Hirata, H., Suster, M.L., Kawakami, K., and Higashijima, S.I. (2013). Transgenic tools to characterize neuronal properties of discrete populations of zebrafish neurons. Dev. 140, 3927-3931.

82. Wen, L., Wei, W., Gu, W., Huang, P., Ren, X., Zhang, Z., Zhu, Z., Lin, S., and Zhang, B. (2008). Visualization of monoaminergic neurons and neurotoxicity of MPTP in live transgenic zebrafish. Dev. Biol. 314, 84-92.

83. Hibi, M., and Shimizu, T. (2012). Development of the cerebellum and cerebellar neural circuits. Dev. Neurobiol. 72, 282-301.

84. Mirat, O., Sternberg, J.R., Severi, K.E., and Wyart, C. (2013). ZebraZoom: an automated program for highthroughput behavioral analysis and categorization. Front Neural Circuits 7, 107.

85. Marques, J.C., Lackner, S., Félix, R., and Orger, M.B. (2018). Structure of the Zebrafish Locomotor Repertoire Revealed with Unsupervised Behavioral Clustering. Curr. Biol., 1-15.

86. Deliagina, T.G., Zelenin, P. V., and Orlovsky, G.N. (2002). Encoding and decoding of reticulospinal commands. Brain Res Brain Res Rev 40, 166-177.

87. Pavlova, E.L., and Deliagina, T.G. (2002). Responses of reticulospinal neurons in intact lamprey to pitch tilt. J. Neurophysiol. 88, 1136-1146.

88. Hale, M.E. (2014). Developmental change in the function of movement systems: Transition of the pectoral fins between respiratory and locomotor roles in zebrafish. Integr. Comp. Biol. 54, 238-249.

89. Apps, R., and Garwicz, M. (2005). Anatomical and physiological foundations of cerebellar information processing. Nat. Rev. Neurosci. 6, 297-311.

90. Ruigrok, T.J.H. (2013). Cerebellar influences on descending spinal motor systems. In Handbook of the Cerebellum and Cerebellar Disorders.

91. Kimura, Y., Satou, C., Fujioka, S., Shoji, W., Umeda, K., Ishizuka, T., Yawo, H., and Higashijima, S.I. (2013). Hindbrain V2a neurons in the excitation of spinal locomotor circuits during zebrafish swimming. Curr. Biol. 23, 843-849.

92. Usseglio, G., Gatier, E., Heuzé, A., Hérent, C., and Bouvier, J. (2020). Control of Orienting Movements and Locomotion by Projection-Defined Subsets of Brainstem V2a Neurons. Curr. Biol., 1-17.

93. Cregg, J.M., Leiras, R., Montalant, A., Wanken, P., Wickersham, I.R., and Kiehn, O. (2020). Brainstem neurons that command mammalian locomotor asymmetries. Nat. Neurosci. 23, 730-740.

94. Frank, K., and Fuortes, M.G. (1956). Stimulation of spinal motoneurones with intracellular electrodes. J. Physiol., 451-470. 
95. Eccles, J.C., Schmidt, R., and Willis, W.D. (1963). Pharmacological studies on presynaptic inhibition. J. Physiol.

96. Eccles, J.C., Kostyuk, P.G., and Schmidt, R.F. (1962). Presynaptic inhibition of the central actions of flexor reflex afferents. J. Physiol.

97. Eccles, J.C., Eccles, R.M., and Magni, F. (1961). Central inhibitory action attributable to presynaptic depolarization produced by muscle afferent volleys. J. Physiol. 159, 147-166.

98. Davies, C.H., Davies, S.N., and Collingridge, G.L. (1990). Paired-pulse depression of monosynaptic GABAmediated inhibitory postsynaptic responses in rat hippocampus. J. Physiol., 513-531.

99. Thompson, S.M., Capogna, M., and Scanziani, M. (1993). Presynaptic inhibition in the hippocampus. Trends Neurosci., 222.

100. Calabresi, P., Mercuri, N.B., De Murtas, M., and Bernardi, G. (1990). Endogenous GABA mediates presynaptic inhibition of spontaneous and evoked excitatory synaptic potentials in the rat neostriatum. Neurosci. Lett. 118, 99-102.

101. Kishore, S., Cadoff, E.B., Agha, M.A., and McLean, D.L. (2020). Orderly compartmental mapping of premotor inhibition in the developing zebrafish spinal cord. Science (80-. ). 370, 431 LP - 436.

102. Stoeckel, M.E., Uhl-Bronner, S., Hugel, S., Veinante, P., Klein, M.J., Mutterer, J., Freund-Mercier, M.J., and Schlichter, R. (2003). Cerebrospinal fluid-contacting neurons in the rat spinal cord, a Y-aminobutyric acidergic system expressing the P2X2 subunit of purinergic receptors, PSA-NCAM, and GAP-43 immunoreactivities: Light and electron microscopic study. J. Comp. Neurol.

103. Watson, C., and Harvey, A. (2009). Projections from the Brain to the Spinal Cord. In The Spinal Cord (Chapter 11), pp. 168-179.

104. Nair, A., Azatian, G., and McHenry, M.J. (2015). The kinematics of directional control in the fast start of zebrafish larvae. J. Exp. Biol. 218, 3996-4004.

105. Bishop, B.H., Spence-Chorman, N., and Gahtan, E. (2016). Three-dimensional motion tracking reveals a diving component to visual and auditory escape swims in zebrafish larvae. J Exp Biol 219, 3981-3987.

106. Metcalfe, W.K., Mendelson, B., and Kimmel, C.B. (1986). Segmental homologies among reticulospinal neurons in the hindbrain of the zebrafish larva. J. Comp. Neurol. 251, 147-159.

107. Deliagina, T.G., and Fagerstedt, P. (2000). Responses of reticulospinal neurons in intact lamprey to vestibular and visual inputs. J. Neurophysiol. 83, 864-878.

108. Deliagina, T.G., Beloozerova, I.N., Orlovsky, G.N., and Zelenin, P. V. (2014). Contribution of supraspinal systems to generation of automatic postural responses. Front. Integr. Neurosci. 8, 1-20.

109. Maldonado, H. (1970). The deimatic reaction in the praying mantis Stagmatoptera biocellata. $\mathrm{Z}$. Vgl. Physiol. 68, 60-71.

110. Randlett, O., Haesemeyer, M., Forkin, G., Shoenhard, H., Schier, A.F., Engert, F., and Granato, M. (2019). Distributed Plasticity Drives Visual Habituation Learning in Larval Zebrafish. Curr. Biol. 29, 1337-1345.e4.

111. Voesenek, C.J., Pieters, R.P.M., Muijres, F.T., and van Leeuwen, J.L. (2019). Reorientation and propulsion in fast-starting zebrafish larvae: An inverse dynamics analysis. J. Exp. Biol. 222.

112. Kimura, Y., and Higashijima, S. ichi (2019). Regulation of locomotor speed and selection of active sets of neurons by $\mathrm{V} 1$ neurons. Nat. Commun. 


\section{STAR METHODS}

\section{RESOURCE AVAILABILITY}

\section{Lead Contact}

Further information and requests for resources should be directed to and will be fulfilled by the Lead Contact, Claire Wyart (claire.wyart@icm-institute.org).

\section{Materials Availability}

Transgenic fish lines generated in this study are available upon request to the lead contact in ICM.

\section{Data and Code Availability}

The datasets and code generated during this study are available at https://github.com/MingYuedanio/Wu-et-al-2020 .

\section{EXPERIMENTAL MODEL AND SUBJECT DETAILS}

Animal handling and procedures were validated by the Paris Brain Institute (ICM) and the French National Ethics Committee (Comité National de Réflexion Ethique sur l'Expérimentation Animale; APAFIS \# 2018071217081175) in agreement with EU legislation. All experiments were performed on Danio rerio larvae of $A B$, Tüpfel long fin (TL) or nacre background. Adult zebrafish were reared at a maximal density of 8 animals per liter in a $14 / 10 \mathrm{hr}$ light/dark cycle environment at $28.5^{\circ} \mathrm{C}$. Larvae were raised at the same conditions. Experiments were performed at $20^{\circ} \mathrm{C}$ or $29^{\circ} \mathrm{C}$ on 3 to 6 days post fertilization (dpf) larvae based on the protocol of each experiment. All transgenic lines used in this study are detailed in Table 1. 
Wu et al. revised manuscript to Current Biology

\begin{tabular}{|c|c|c|c|}
\hline Transgenic name & Allele name & Labelling & Original publication \\
\hline $\operatorname{Tg}(p k d 2 / 1: G A L 4)$ & icm10 & CSF-cNs & Fidelin et al., 2015 [1] \\
\hline $\operatorname{Tg}(p k d 2 / 1: \operatorname{tag} R F P)$ & icm17 & CSF-cNs & $\begin{array}{l}\text { Böhm, Prendergast et al, } \\
2016 \text { [2] }\end{array}$ \\
\hline $\operatorname{Tg}(p k d 2 / 1: e G F P)$ & icm56 & CSF-cNs & This paper \\
\hline $\operatorname{Tg}($ UAS:ChR2-mCherry) & s1985t & NA & Schoonheim et al., 2010 [3] \\
\hline $\operatorname{Tg}(U A S: C h R 2-Y F P)$ & icm11 & NA & Fidelin et al., 2015 [1] \\
\hline $\operatorname{Tg}($ parga:GFP) & $m n 2 E t$ & Primary Motor Neurons & Balciunas et al.,2004 [4] \\
\hline $\operatorname{Tg}(T o l 056: G F P)$ & zf206Et & CoLo interneurons & Satou et al., 2009 [5] \\
\hline$T g(v m a t 2: G F P)$ & zf710 & Monoaminergic neurons & Wen et al, 2008 [6] \\
\hline $\begin{array}{l}\text { Tg(zCREST2- } \\
\text { hsp70:GFP) }\end{array}$ & $r w 011 a$ & $\begin{array}{c}\text { Motor neurons } \\
\text { innervating abductor fin } \\
\text { muscles }\end{array}$ & Uemura et al., 2005 [7] \\
\hline $\operatorname{Tg}($ gad1b:GFP) & $n n s 25$ & GABAergic neurons & Satou et al., 2013 [8] \\
\hline$T g(g l y t 2: G F P)$ & nns23 & Glycinergic neurons & Satou et al., 2013 [8] \\
\hline $\begin{array}{c}\text { Tg(vglut2a:loxP-DsRed- } \\
\text { loxP-GFP) }\end{array}$ & $z f 139$ & Glutamatergic neurons & Koyama et al., 2011 [9] \\
\hline $\operatorname{Tg}(\operatorname{ch} \times 10: G F P)$ & nns1 & V2a interneurons & Kimura et al., 2006 [10] \\
\hline $\operatorname{Tg}(U A S: G F P)$ & - & NA & Kawakami, 2007 [11] \\
\hline $\operatorname{Tg}(\alpha$-actin:GAL4) & icm51 & Axial muscles & This paper \\
\hline
\end{tabular}

\section{METHOD DETAILS}

\section{Generation of stable transgenic lines}

To generate a Tol2 vector driving GAL4 under the control of a-actin regulatory elements, we used Gateway recombination-based cloning (Thermo Fisher, 11791020) using p5E$\alpha$-actin (the a-actin promoter was a kind gift from Prof. Philip W. Ingham, Nanyang Technological University, Singapore), pME-GAL4, and p3E-poly(A) into pDestpA2. The 
resulting vector was injected into $T g(U A S: C h R 2-Y F P)$ at $30 \mathrm{ng} / \mu \mathrm{L}$ with $35 \mathrm{ng} / \mu \mathrm{L}$ Tol2 transposase to generate germline transgenics as previously described (Fisher et al., 2006; Kwan et al., 2007). Transgenic founder fish Tg(a-actin:GAL4) ${ }^{i c m 51}$ were screened based on transactivation of the transgene when crossed with various UAS lines. The $T g(p k d 2 / 1: e G F P)^{i c m 56}$ has been generated with a similar approach. The final three-way gateway reaction used the p5E-pkd2I1, pME-eGFP and p5E-pkd2l1-intron plasmids into pDestpA2. The resulting vector was injected into $A B$ wild type fish at $30 \mathrm{ng} / \mu \mathrm{L}$ with 35 $\mathrm{ng} / \mu \mathrm{L}$ Tol2 transposase to generate germline transgenics. Transgenic founder fish $T g(p k d 2 / 1: e G F P)^{i c m 56}$ were screened based on GFP expression showing a faithful $p k d 2 / 1$ expression pattern.

\section{Injection of DNA constructs}

To label individual CSF-cN and study its projection on specific targets, we injected $2 \mathrm{~nL}$ of $10 \mathrm{ng} / \mathrm{\mu L}$ plasmid UAS:synaptophysin-mCherry (or UAS:Synaptophysin(GGGS) ${ }_{3}-$ $m$ Cherry-pA) in the single cell-stage embryos of double transgenic lines: Tg(pkd2/1:GAL4;Tol056:GFP) and Tg(pkd2/1:GAL4;chx10:GFP). To sparsely express $m$ Cherry-zGephyrin in Chx $10^{+}$reticulospinal neurons, we injected $10 \mathrm{ng} / \mu \mathrm{L}$ plasmid (pT2MUAS:mCherry-zGephyrin-aP1) in the single cell-stage embryos of double transgenic line $T g(p k d 2 / 1: e G F P ; c h \times 10: G A L 4)$. The injected embryos were screened for sparse labeling at 2-3 dpf.

\section{Live imaging and anatomical analysis}

All images for anatomical analysis were acquired by a confocal microscope combing an upright microscope (Examiner Z1, Zeiss), a spinning disk head (CSU-W1, Yokogawa) and a laser light source (LasterStack, 3i Intelligent Imaging Innovations). The different lines labelling specific neuronal populations were crossed with $T g(p k d 2 / 1: T a g R F P)$, Tg(pkd2/1:GAL4;UAS:FP) to examine the anatomical connections between CSF-cNs and targeted neurons. Larvae that were not nacre background were treated with $4.5 \mu \mathrm{g} / \mathrm{mL}$ PTU (Sigma-Aldrich) to inhibit melanogenesis from 12 hours post fertilization (hpf). 4-5 
dpf larvae were anesthetized with $0.02 \%$ MS-222 and mounted on their side or upright position in 1.5\% agarose. Images were acquired using a 20X/1.0 DIC D=0.17 M27 75mm (Zeiss, no:421452-9880-000) or a 40X/1.0 DIC M27 (Zeiss, no.: 421462-9900-000). Zprojection stacks from lateral or dorsal view were acquired with a step of $0.53 \mu \mathrm{m}$ or $1 \mu \mathrm{m}$ depth using Slidebook software 6.0 (3i, Intelligent Imaging Innovations) and reconstructed using Fiji [12] (http://fiji.sc/Fiii).

\section{Whole-cell electrophysiology}

Electrophysiological recording was performed at room temperature $\left(22^{\circ} \mathrm{C}-25^{\circ} \mathrm{C}\right)$ on $4-5$ dpf larvae. Zebrafish larvae were firstly anesthetized with $0.02 \% \mathrm{MS}-222$ and mounted in $1.5 \%$ agarose before being paralyzed by injecting $0.5 \mathrm{nl}$ of $0.5 \mathrm{mM}$ a-bungarotoxin (Tocris) into the ventral axial musculature. Larvae were then unmounted from the agarose and pinned to a Sylgard-coated recording chamber (Sylgard 184; Dow Corning) through the notochord with sharp tungsten pins. External bath recording solution contained the following: $134 \mathrm{mM} \mathrm{NaCl}, 2.9 \mathrm{mM} \mathrm{KCl}, 2.1 \mathrm{mM} \mathrm{CaCl} 2-\mathrm{H}_{2} \mathrm{O}, 1.2 \mathrm{mM} \mathrm{MgCl}_{2}, 10 \mathrm{mM}$ glucose, and $10 \mathrm{mM}$ HEPES, with the $\mathrm{pH}$ adjusted to 7.4 and osmolarity to $290 \mathrm{mOsm}$. The head was then fixed to upright position by pinning another two tungsten pins through the otic vesicle or the cartage. The skin was removed to expose the hindbrain region and the $\mathrm{Oc} / \mathrm{Pec}$ motor column was exposed by gently suctioning away the dorsal brain tissue with a glass pipette under fluorescent microscope. Recording electrodes were designed to reach a resistance of 10-16 M $\Omega$ with capillary glass (1B150F-4, WPI) on a horizontal puller (P1000; Sutter Instrument). Internal solution contained the following: $115 \mathrm{mM} \mathrm{K}$ gluconate, $15 \mathrm{mM} \mathrm{KCl}, 2 \mathrm{mM} \mathrm{MgCl}$, $4 \mathrm{mM}$ Mg-ATP, EGTA 0.5, $10 \mathrm{mM}$ HEPES, with the $\mathrm{pH}$ adjusted to 7.2 and osmolarity to $290 \mathrm{mOsm}$, supplemented with Alexa 647 at $4 \mathrm{mM}$ (Life Technologies) or rhodamine (Sigma-Aldrich) diluted to $0.1 \%$ (for reconstruction the recorded cell). Positive pressure $(45 \mathrm{mmHg})$ was applied to the recording electrode via a pneumatic transducer (Fluke Biomedical; DPM1B). Cells were chosen based on their GFP expression and axon arborization of CSF-cNs expressing ChR2-mCherry. Image stacks were taken before and after the recording with a confocal microscope combing an upright microscope (Examiner Z1, Zeiss), a spinning disk head (CSU-X1, Yokogawa) and 
a laser light source (LasterStack, 3i Intelligent Imaging Innovations). Once the electrode was driven to push against the target neuron and a dip formed at the soma surface, the positive pressure was removed to form the gigaseal. A brief suction and/or electrical

shock was applied to get the whole cell recording. AP5 (Tocris) and CNQX (Tocris) were bath applied at 10-20 $\mu \mathrm{M}$ final concentrations to block the excitatory synaptic inputs. An Axopatch 700B amplifier, a Digidata series 1440A Digitizer and pClamp 10.3 software (Axon Instruments, Molecular Devices, San Jose, California, USA) were used to acquire the electrophysiological data at a sampling rate of $50 \mathrm{kHz}$ and low pass filtered at $10 \mathrm{kHz}$. A blue LED (UHP-Mic-LED-460, Prizmatix) was controlled by Clampex 10.3 to generate pulsed light at different frequencies to activate the ChR2 through the condenser in a whole-field manner. Data were analyzed offline with Clampfit 10.7 (Molecular Devices, San Jose, California, USA), Excel 2016 (Microsoft), and Matlab R2018b (The MathWorks Inc., USA). The light-induced inhibitory postsynaptic currents (IPSCs) were calculated as the difference between the baseline before the optical stimulation and the peak signal in a 200 ms window after the stimulation.

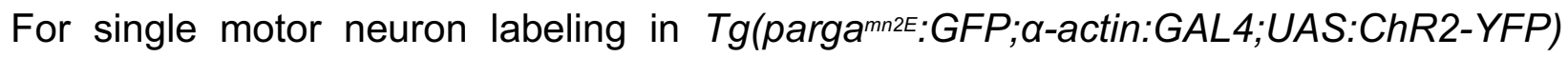
larvae, rhodamine dye loading was performed as described above for the whole-cell patch. Basic firing pattern and postsynaptic input currents were recorded for 5-10 minutes to confirm the cell was alive. Image stacks were taken before and after the recording for cell reconstruction. After recording, the larva was freed from tungsten pins and embedded upside-down in agarose to check the target muscles of the motor neuron.

\section{Two-photon mediated cell ablation}

To guarantee the transparency of larvae for two-photon cell ablation at $4 \mathrm{dpf}$ and to facilitate the detection of larvae by the tracking algorithm at $6 \mathrm{dpf}, A B$ larvae were treated with $4.5 \mu \mathrm{g} / \mathrm{mL}$ PTU to inhibit melanogenesis from $12 \mathrm{hpf}$ until $4 \mathrm{dpf}$. After cell ablation, larvae were let to recover in system water for two days to allow pigmentation. Cell ablation was performed at $20^{\circ} \mathrm{C}$ using a two-photon laser microscope (2p-vivo, Intelligent Imaging Innovations, Inc., Denver, USA). Larvae were anaesthetized with $0.02 \% \mathrm{MS}-222$ and embedded on their side in $1.5 \%$ low melting agarose. The CSF-cNs are evenly distributed 
along the ventral spinal cord. A scanning line equal to the length of soma was drawn for each cell on the same Z-plane. High power laser $(800 \mathrm{~nm},>100 \mathrm{~mW})$ pulses were delivered through a 20x objective lens (Zeiss, no:421452-9880-000), to run 20-30 line scans to the targeted cell. Power and scanning repeats were determined by observing a focal increase in fluorescence, indicative of a successful ablation. 60-70 neurons within six segments of either the rostralmost or the caudalmost spinal cord were targeted across multiple $Z$ planes. Larvae were then freed and returned to system water to recover until the behavioral test at $6 \mathrm{dpf}$. The efficiency of ablation was confirmed by imaging each fish after the behavioral assay.

\section{Behavioral recordings and analysis}

\section{Behavior test}

Behavior tests were performed at $27^{\circ} \mathrm{C}-29^{\circ} \mathrm{C}$. The behavior setup was adapted from the one previously described $[2,13,14]$. To record the locomotor behaviors, an Arduino Uno board (Arduino) was designed to trigger a high-speed camera (acA2000-340km with focus area up to $2000 \times 1088$ pixels, Basler, Ahrensburg, Germany) and a video recording software (Hiris, R\&D vision, France). Four circular swim arenas (with a $2.2 \mathrm{~cm}$ inner diameter, $2 \mathrm{~mL}$ system water filled with an estimated height of $525 \mu \mathrm{m}$ ) with one larva in each were placed above a plexiglass plate on which 2 speakers (Monacor, 10W) were attached. A flat LED plate (R\&D vision, France) with a polarized optical filter was placed below the transparent plexiglass to provide homogeneous field illumination. Fish were allowed to acclimate for $10 \mathrm{~min}$. The exploratory locomotion was recorded for $5 \mathrm{~min}$ at $160 \mathrm{fps}$, then the video software was switched to record the acoustic stimulus evoked escape behaviors at $650 \mathrm{fps}$ for one second. To trigger escape behaviors, a $500 \mathrm{~Hz}, 5-$ ms sine wave stimulus was delivered through a class D amplifier (Adafruit, MAX9744) over the two speakers at maximum volume, $200 \mathrm{~ms}$ after the onset of high speed recording. Each larva was subjected to ten stimuli, with 3 min inter-trial interval. 


\section{Analysis of behavior kinematics}

The raw videos were analyzed using ZebraZoom [2,13,14] (https://zebrazoom.org/), and Matlab R2018b (The MathWorks Inc. USA). The following escape responses parameters were calculated from the tail kinematics: latency (interval between the stimulus and onset of the tail bend), bout duration, (time of the detected movement), C-bend amplitude (absolute peak amplitude of the first tail bend), time to peak of C-bend (interval between the onset of tail bend and peak amplitude of C-bend), number of oscillations (1/2 of the number of tail angle peaks in one escape), averaged TBF (mTBF; number of oscillations / bout duration). The interval between C-bend and counter bend was used to define the starting tail beating frequency $\left(\mathrm{TBF}_{1}: 1 / 2\right.$ of the inverse of interval between $\mathrm{C}$-bend and counter bend). The trajectory of the head was used to define the distance traveled and speed (bout distance / bout duration). Visual inspection of escape videos and tracked tail bending traces was performed to exclude bouts that were not escapes (responses happening before or $50 \mathrm{~ms}$ after the stimulus) or erroneously tracked.

\section{Analysis of rolling behavior}

A deep learning method was designed to obtain automated measurements of rolling behavior and to analyze the onset of postural defects. The deep learning neural network was based on the module using the pre-trained neural network architecture MobileNet V2 (depth multiplier 1.00) [19] and its feature vectors of images obtained by training on ImageNet (ILSVRC-2012-CLS) from TensorFlowHub (Google, https://tfhub.dev/). A transfer learning strategy was employed. A sample of frames from 12 different videos (48 bouts, 30384 frames) were manually classified as 'ROLL' (1.99\%), 'NO ROLL' (95.73\%), or 'AMBIGUOUS' $(2.27 \%)$ and were used to retrain the neural network. Cross-validation was used to test the accuracy of the trained deep learning classifier (the first time by excluding the first video and testing on that first video, the second time by excluding the second video and testing on that second video, etc.). For each of the 12 videos, a true positive rate was calculated as: number of frames 'correctly' classified as 'ROLL' by the classifier / number of frames manually classified as 'ROLL' 
The general true positive rate was the weighted average of those 12 true positive rates. After the training, a true positive rate of $96.86 \%$ and a false positive rate of $0.62 \%$ were reached. Finally, each frame of the recorded videos was processed and assigned a rolling probability by the deep learning classifier. A 'rolling event' was defined if the rolling probability of a frame is higher than $80 \%$. The total rolling duration was calculated as the total time the rolling event happened. A long rolling event was defined if the larva showed sustained rolling event for more than $10 \mathrm{~ms}$.

\section{QUANTIFICATION AND STATISTICAL ANALYSIS}

All values are shown as mean \pm standard error of the mean (SEM). Data are presented as a mean of per larva. The violin plot shows the estimated distribution as well as the mean (red line) and median (black line). In all figures, $* P<0.05$, ** $P<0.01$, *** $P<$ 0.001 , and $* * * * P<0.0001$.

\section{Analysis of the whole-cell patch electrophysiology}

Current events occurred within $10 \mathrm{~ms}$ delay of the light pulse were analyzed for recorded cells. The delay, amplitude, and rise time of the first light pulse induced current event are calculated. In some instances, motor neurons receive inputs with distinct delays originating from more than one CSF-cNs that spikes at different times and led to more than one IPSCs in the same neuron recorded. We could not analyze the time decay in these cases. Summary data are presented as mean \pm SEM. Comparisons of amplitude of IPSCs between light on and light off trials were performed using a Student's paired $t$ test (GraphPad Prism, 8.0.2). A value of $P<0.05$ was considered significant.

\section{Analysis of the kinematics of escape responses}

The kinematics of escape responses was analyzed using $R$, version 3.5 .2 [20] (http://cran.rproject.org/). For longitudinal data across trials (repetitions within the same fish), the comparisons between treatments (control, rostral ablation and caudal ablation) 
were performed using linear mixed models (LMMs) with fixed effects for treatments and trials (1-10) and random effects for animal-specific variation (fish numbers nested within clutches). LMM was fitted for each parameter using the function Imer in the Ime4 package. When necessary, the data were either log or square root transformed prior to the modeling to better match the model assumptions (normality and homoscedasticity of residuals). The significance for main effects of treatment, trial and their interaction were then evaluated with the Anova function in the car package using Type II Wald chi-square tests. As multiple parameters $(\mathrm{N}=8)$ from the same behavior tracking data were analyzed, the significance level for ANOVA was adjusted with Bonferroni correction to $P<0.05$ / N. Post hoc pairwise comparisons between the three treatments were then tested using the emmeans package with the Tukey's method for multiplicity adjustment and a significance level of adjusted $P<0.05$.

\section{Analysis of the rolling events during escape responses}

Data were presented as mean per larva and plotted with violin plot. The two parameters related to postural defects (rolling duration and number of long rolling events) were analyzed with the procedure described above for the kinematics parameters. However, for the number of long rolling, count data were fitted by a Poisson generalized linear mixed model (GLMM) with a square root link using the glmer function in the Ime4 package. Bonferroni correction was applied with $N=2$. This analysis was performed over the whole ten trials (1-10), and then performed over the first five trials (1-5) and the last five trials (6-10). For the onset of rollovers, data were presented as values for each escape with a box plot. Significance for the effect of treatment was tested with ordinary one-way ANOVA (GraphPad Prism 8.0.2).

\section{References}

1. Fidelin, K., Djenoune, L., Stokes, C., Prendergast, A., Gomez, J., Baradel, A., Del Bene, F., and Wyart, C. (2015). State-dependent modulation of locomotion by GABAergic spinal sensory neurons. Curr. Biol. 25, 3035-3047.

2. Böhm, U.L., Prendergast, A., Djenoune, L., Figueiredo, S.N., Gomez, J., Stokes, C., Kaiser, S., Suster, M., Kawakami, K., Charpentier, M., et al. (2016). CSF-contacting neurons regulate locomotion by relaying mechanical stimuli to spinal circuits. Nat. Commun. 7, 1-8.

3. Schoonheim, P.J., Arrenberg, A.B., Del Bene, F., and Baier, H. (2010). Optogenetic localization and genetic perturbation of saccade-generating neurons in Zebrafish. J. Neurosci. 30, 7111-7120. 
4. Balciunas, D., Davidson, A.E., Sivasubbu, S., Hermanson, S.B., Welle, Z., and Ekker, S.C. (2004). Enhancer trapping in zebrafish using the Sleeping Beauty transposon. BMC Genomics 5, 62.

5. Satou, C., Kimura, Y., Kohashi, T., Horikawa, K., Takeda, H., Oda, Y., and Higashijima, S. (2009). Functional role of a specialized class of spinal commissural inhibitory neurons during fast escapes in zebrafish. J Neurosci 29, 67806793.

6. Wen, L., Wei, W., Gu, W., Huang, P., Ren, X., Zhang, Z., Zhu, Z., Lin, S., and Zhang, B. (2008). Visualization of monoaminergic neurons and neurotoxicity of MPTP in live transgenic zebrafish. Dev. Biol. 314, 84-92.

7. Uemura, O., Okada, Y., Ando, H., Guedj, M., Higashijima, S.I., Shimazaki, T., Chino, N., Okano, H., and Okamoto, H. (2005). Comparative functional genomics revealed conservation and diversification of three enhancers of the isl1 gene for motor and sensory neuron-specific expression. Dev. Biol. 278, 587-606.

8. Satou, C., Kimura, Y., Hirata, H., Suster, M.L., Kawakami, K., and Higashijima, S.I. (2013). Transgenic tools to characterize neuronal properties of discrete populations of zebrafish neurons. Dev. 140, 3927-3931.

9. Koyama, M., Kinkhabwala, A., Satou, C., Higashijima, S., and Fetcho, J. (2011). Mapping a sensory-motor network onto a structural and functional ground plan in the hindbrain. Proc Natl Acad Sci U S A 108, 1170-1175.

10. Kimura, Y., Okamura, Y., and Higashijima, S. (2006). alx, a zebrafish homolog of Chx10, marks ipsilateral descending excitatory interneurons that participate in the regulation of spinal locomotor circuits. J Neurosci 26, 56845697.

11. Kawakami, K. (2007). Tol2: A versatile gene transfer vector in vertebrates. Genome Biol. 8, 1-10.

12. Schindelin, J., Arganda-Carreras, I., Frise, E., Kaynig, V., Longair, M., Pietzsch, T., Preibisch, S., Rueden, C., Saalfeld, S., Schmid, B., et al. (2012). Fiji: An open-source platform for biological-image analysis. Nat. Methods 9 , 676-682.

13. Knafo, S., Fidelin, K., Prendergast, A., Tseng, P.B.E.B., Parrin, A., Dickey, C., Böhm, U.L., Figueiredo, S.N., Thouvenin, O., Pascal-Moussellard, H., et al. (2017). Mechanosensory neurons control the timing of spinal microcircuit selection during locomotion. Elife 6.

14. Mirat, O., Sternberg, J.R., Severi, K.E., and Wyart, C. (2013). ZebraZoom: an automated program for high-throughput behavioral analysis and categorization. Front Neural Circuits 7, 107.

19. Mark Sandler, Andrew Howard, Menglong Zhu, Andrey Zhmoginov, Liang-Chieh Chen. (2019). MobileNetV2: Inverted Residuals and Linear Bottlenecks. arXiv:1801.04381

20. R Core Team (2018). R: A language and environment for statistical computing. R Foundation for Statistical Computing, Vienna, Austria. URL https://www.R-project.org/. 